\title{
N-Terminal Motifs in Some Plant Disease Resistance Proteins Function in Membrane Attachment and Contribute to Disease Resistance
}

\author{
Daigo Takemoto, ${ }^{1}$ Maryam Rafiqi, ${ }^{1}$ Ursula Hurley, ${ }^{1}$ Greg J. Lawrence, ${ }^{2}$ Maud Bernoux, ${ }^{2}$ \\ Adrienne R. Hardham, ${ }^{1}$ Jeffrey G. Ellis, ${ }^{2}$ Peter N. Dodds, ${ }^{2}$ and David A. Jones ${ }^{1}$ \\ ${ }^{1}$ Plant Science Division, Research School of Biology, The Australian National University, Canberra ACT 0200, Australia; \\ ${ }^{2}$ CSIRO Plant Industry, GPO Box 1600, Canberra ACT 2601, Australia
}

Submitted 23 November 2010. Accepted 26 October 2011.

To investigate the role of $\mathrm{N}$-terminal domains of plant disease resistance proteins in membrane targeting, the $\mathbf{N}$ termini of a number of Arabidopsis and flax disease resistance proteins were fused to green fluorescent protein (GFP) and the fusion proteins localized in planta using confocal microscopy. The $\mathbf{N}$ termini of the Arabidopsis RPP1-WsB and RPS5 resistance proteins and the PBS1 protein, which is required for RPS5 resistance, targeted GFP to the plasma membrane, and mutation of predicted myristoylation and potential palmitoylation sites resulted in a shift to nucleocytosolic localization. The $\mathrm{N}$-terminal domain of the membrane-attached Arabidopsis RPS2 resistance protein was targeted incompletely to the plasma membrane. In contrast, the N-terminal domains of the Arabidopsis RPP1-WsA and flax L6 and $M$ resistance proteins, which carry predicted signal anchors, were targeted to the endomembrane system, RPP1-WsA to the endoplasmic reticulum and the Golgi apparatus, L6 to the Golgi apparatus, and $M$ to the tonoplast. Full-length L6 was also targeted to the Golgi apparatus. Site-directed mutagenesis of six nonconserved amino acid residues in the signal anchor domains of L6 and $M$ was used to change the localization of the $L 6$ N-terminal fusion protein to that of $M$ and vice versa, showing that these residues control the targeting specificity of the signal anchor. Replacement of the signal anchor domain of L6 by that of M did not affect L6 protein accumulation or resistance against flax rust expressing AvrL567 but removal of the signal anchor domain reduced L6 protein accumulation and $\mathrm{L6}$ resistance, suggesting that membrane attachment is required to stabilize the $\mathrm{L} 6$ protein.

Plants have the ability to detect and resist infection by most plant pathogens but each species of plant falls victim to a range of plant pathogens that have evolved effectors to overcome basal

D. Takemoto and M. Rafiqi contributed equally to the work described in this article.

Current address for D. Takemoto: Plant Pathology Laboratory, Graduate School of Bioagricultural Sciences, Nagoya University, Chikusa, Nagoya 464-8601, Japan.

Corresponding author: D. Jones: E-mail: david.jones@ anu.edu.au

* The $\boldsymbol{e}$-Xtra logo stands for "electronic extra" and indicates that supplementary text, four supplementary figures, and two supplementary tables are published online. Also, Figures 1, 2, 4, 6, 7, 8, and 10 appear in color online. plant defenses (Dodds and Rathjen 2010; Jones and Dangl 2006). Plants, in turn, have evolved specialized defenses controlled by disease resistance genes that confer the ability to recognize and respond to pathogen effectors. Many plant disease resistance genes have now been isolated, with most encoding cytosolic nucleotide-binding leucine-rich repeat (NB-LRR) proteins (Rafiqi et al. 2009). These proteins can be subdivided according to their N-terminal domain into coiled-coil (CC-NBLRR) and Toll/interleukin-1 receptor homology (TIR-NB-LRR) subclasses. Although sharing broad structural similarities, many NB-LRR proteins also show unique structural variations that are important for their function. Examples include $\mathrm{N}$ - and C-terminal extensions, duplications of domains, additional structural domains, and potential subcellular-localization signals.

Several resistance proteins are predicted to have N-terminal membrane-localization signals but membrane attachment has only been confirmed experimentally for Arabidopsis RPP1WsA, which confers resistance to the downy mildew pathogen Hyaloperonospora arabidopsidis. RPP1-WsA has a predicted N-terminal signal anchor sequence (Botella et al. 1998) that targets RPP1-WsA to the membrane of the endoplasmic reticulum (ER) or the Golgi apparatus (GA) (Weaver et al. 2006). Signal anchors, like signal peptides, have hydrophobic N-terminal sequences that direct nascent polypeptides to the ER where they are inserted into the ER membrane but, unlike signal peptides, are not removed by signal peptidase. Therefore, the entire protein remains attached to the membrane via the signal anchor with either the $\mathrm{N}$ terminus (only a few amino acids) exposed on the extracytoslic side of the membrane and the $\mathrm{C}$ terminus (the bulk of the protein) on the cytosolic side (type I orientation) or vice versa (type II orientation). Signalanchored proteins, like other transmembrane proteins, can be retained on the ER membrane or directed to other subcellular membranes or the plasma membrane (PM). Remarkably, two RPP1 paralogs, RPP1-WsB and RPP1-WsC, lack this signal but have predicted $\mathrm{N}$-terminal myristoylation and palmitoylation sites that could provide an alternative means of membrane attachment (Botella et al. 1998) (Table 1). The long hydrophobic chains of myristic acid and palmitic acid insert into membrane lipid bilayers, thereby anchoring myristoylated and palmitoylated proteins to the membrane. The Arabidopsis RPS5 protein conferring resistance to the bacterial speck pathogen Pseudomonas syringae also has predicted N-terminal myristoylation and palmitoylation sites (Table 1) and has been shown experimentally to be membrane anchored (Holt et al. 2005); however, its subcellular location has not been determined experimentally and the role of the N-terminal domain of RPS5 
in membrane-attachment has not been tested directly. The flax L6 and M proteins conferring resistance to the flax rust fungus Melampsora lini have predicted N-terminal signal anchor domains (Supplementary Text) but membrane attachment of

Table 1. N-terminal myristoylation and palmitoylation motifs present in a number of resistance (R) and avirulence (Avr) proteins, and proteins mediating the interaction between $\mathrm{R}$ and Avr proteins

\begin{tabular}{lc}
\hline Protein & N-terminal sequence ${ }^{\mathbf{a}}$ \\
\hline RPP1-WsB & MGSAMSLGCS \\
RPP1-WsC & MGSAMSLSCS \\
RPS5 & MGGCESVSLP \\
AvrB & MGCVSSKSTT \\
AvrRpm1 & MGCVSSTSRS \\
AvrPto & MGNICVGGSR \\
AvrPphB & GCASSSGVS \\
PBS1 & MGCFSCFDS \\
Pto & MGSKYSKATN \\
\hline
\end{tabular}

${ }^{a}$ The first 10 amino acids of each protein are shown, except for AvrPphB, in which the first nine amino acids of the mature protein are shown. Glycine and serine residues of the conserved myristoylation motif are highlighted in bold. Cysteine residues, which are potential substrates for palmitoylation, are also highlighted in bold. these proteins has not been tested. In contrast, the Arabidopsis RPM1 and RPS2 proteins, which also confer resistance to $P$. syringae, have no predicted membrane attachment domains, yet both have been shown experimentally to be membrane associated, with RPM1 behaving as a peripheral membrane protein (Boyes et al. 1998) and RPS2 behaving as an integral membrane protein (Axtell and Staskawicz 2003).

Several pathogen effector proteins have also been shown to be associated with the host PM after delivery into the host cell by type III secretion. The P. syringae AvrB, AvrRpm1, and AvrPto proteins have N-terminal myristoylation motifs (Table 1) that direct the attachment of these effector proteins to the PM (Nimchuk et al. 2000; Shan et al. 2000). These avirulence (Avr) proteins also have $\mathrm{N}$-terminal cysteine residues that are potential sites for palmitoylation (Table 1$)$. The $P$. syringae $A v r P p h B$ gene encodes a cysteine protease that undergoes selfcleavage after delivery into plant cells to reveal $\mathrm{N}$-terminal myristoylation and palmitoylation sites that direct the attachment of the protein to the plant PM (Nimchuk et al. 2000) (Table 1). Myristoylation and palmitoylation of AvrPphB have been confirmed recently by Dowen and associates (2009).

Recognition of pathogen effector proteins by plant disease resistance proteins can either be direct or mediated by plant
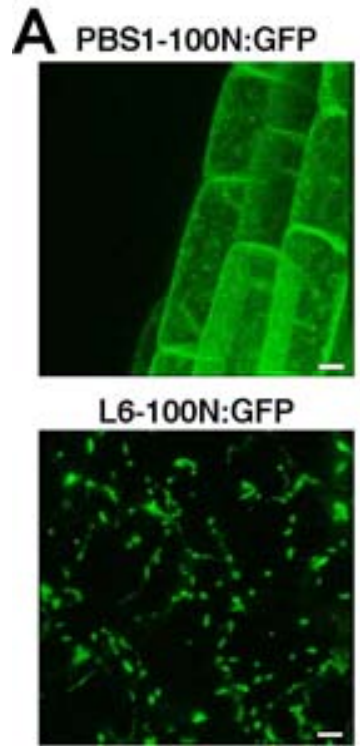

RPP1WsB-100N:GFP

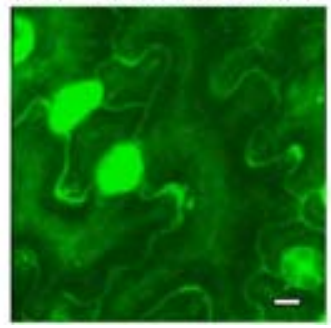

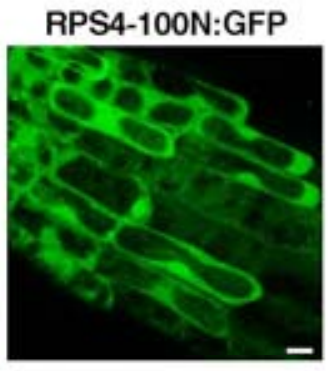

RPP1WSA-100N:GFP

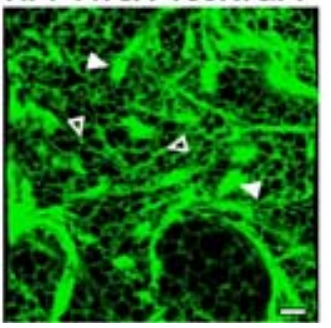

RPS5-100N:GFP

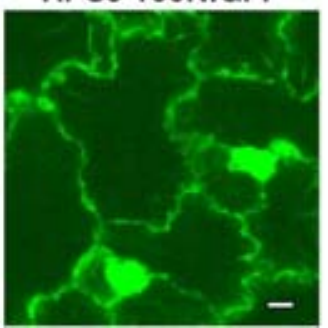

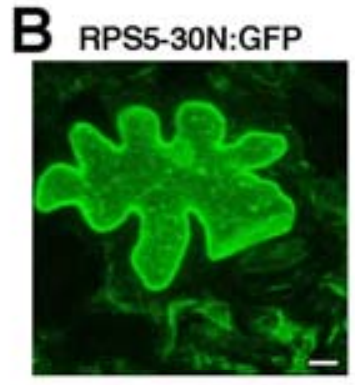
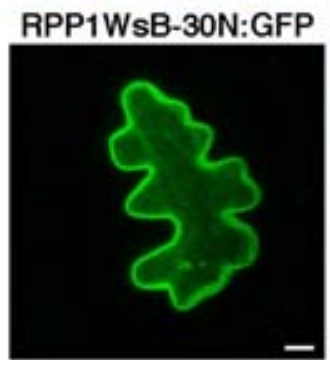

RPM1-30N:GFP

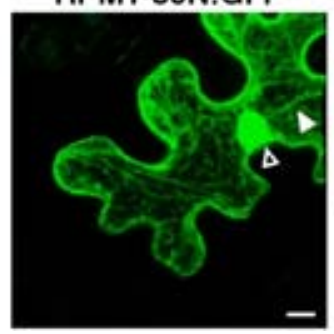

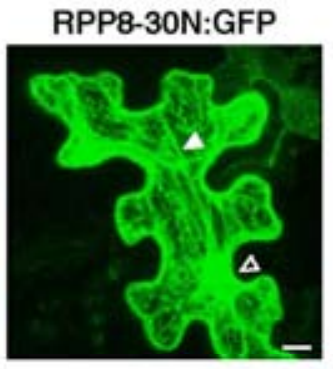

RPS2-30N:GFP

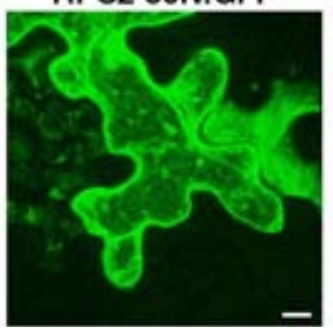

RPS4-30N:GFP

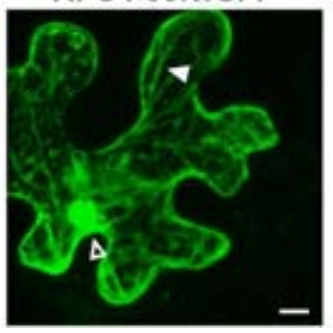

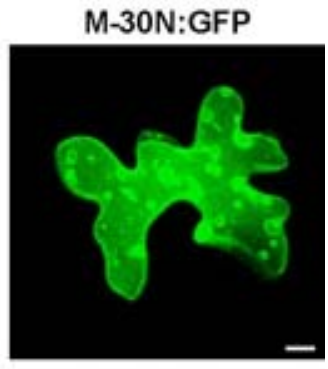

P2-30N:GFP

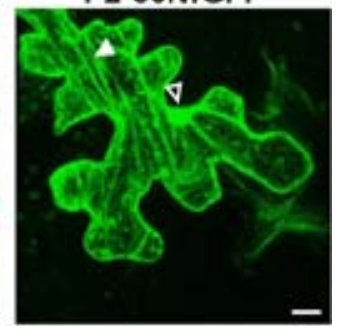

RPP4-30N:GFP

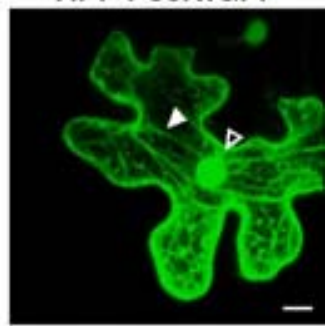

Fig. 1. Examples of localization patterns observed for green fluorescent protein (GFP) fusions to the N-terminal sequences of disease resistance proteins and PBS1 A, in transgenic Arabidopsis or B, following particle bombardment of tobacco leaf epidermal cells. A, Fusion proteins with the N-terminal 100 amino acids (100N) of disease resistance proteins and PBS1 fused to GFP localize to the plasma membrane (PBS1) and to the cytosol and nucleus (RPS4) in roots of transgenic Arabidopsis plants, and to the Golgi apparatus (L6), the endoplasmic reticulum (RPP1-WsA), and plasma membrane (RPP1-WsB and RPS5) in leaf epidermal cells of transgenic Arabidopsis plants. The RPP1-WsA fusion also shows labeling of endoplasmic reticulum (ER) bodies (closed arrowheads) and possibly Golgi apparatus (open arrowheads). Note that that the PBS1 and RPS5 fusion proteins give the plasma membrane a granular appearance that is not evident for RPP1-WsB. This may indicate aggregation of the PBS1 and RPS5 fusions into plasma membrane subdomains (e.g., lipid rafts). Note also the guard cell autofluorescence visible in the RPP1-WsB and RPS5 panels. Bars $=10 \mu \mathrm{m}$. B, Fusion proteins with the N-terminal 30 amino acids (30N) of disease resistance proteins fused to GFP localize to the plasma membrane (RPS5 and RPP1-WsB), to the tonoplast (M), and to the cytosol and nucleus (RPP4, RPP8, RPS4, RPM1, and P2) in epidermal cells of bombarded tobacco leaves. Nucleo-cytosolic localization is characterized by the labeling of cytoplasmic strands (closed arrowheads) and nuclei (open arrowheads). Note that the molecular masses of these fusion proteins fall below the nuclear exclusion limit so that the fusion proteins are able to diffuse freely into the nucleus. The RPS2 fusion shows weak labeling of cytosolic strands but intense labeling at the cell periphery, a pattern of fluorescence consistent with both plasma membrane and nucleo-cytosolic localization. Bars $=10 \mu \mathrm{m}$. 
proteins that are the targets for pathogen effectors. Several plant proteins that mediate the interaction between resistance proteins and pathogen effector proteins have also been shown to be membrane associated. The Arabidopsis RIN4 protein, which mediates the interaction between Arabidopsis RPS 2 and $P$. syringae AvrRpt 2 and between Arabidopsis RPM1 and $P$. syringae AvrRpm1 or AvrB, has a carboxyl-terminal palmitoylation motif that directs its attachment to the PM (Day et al. 2005; Kim et al. 2005; Takemoto and Jones 2005). The Arabidopsis PBS1 protein, which mediates the interaction between Arabidopsis RPS5 and P. syringae AvrPphB (Ade et al. 2007), has a predicted N-terminal myristoylation motif (Swiderski and Innes 2001) but membrane attachment has not been demonstrated. The tomato Pto protein, which mediates the interaction between the tomato Prf resistance protein and $P$. syringae AvrPto or AvrPtoB, has a functional N-terminal myristoylation site but does not seem to be attached to the PM (de Vries et al. 2006). Rather, the N-terminal myristate interacts with Pto itself to repress its kinase activity and is thought to be released from this interaction upon activation of Pto by AvrPto (Andriotis and Rathjen 2006). Whether the exposed myristate then enables Pto to be attached to a membrane after activation by AvrPto is not known.

In this article, we demonstrate membrane attachment for a number of resistance and resistance-associated proteins with predicted N-terminal membrane-attachment domains (myristoylation or palmitoylation sites or signal anchors) using green fluorescent protein (GFP) fusions. These fusions reveal that the various $\mathrm{N}$-terminal membrane attachment domains of these proteins are targeted to different subcellular membranes. We examine the molecular basis for membrane attachment and targeting by site-directed mutagenesis and focus on the functional significance of membrane attachment on resistance gene function in the flax-flax rust interaction.

\section{RESULTS}

The $\mathbf{N}$ termini of some resistance proteins function as membrane-attachment domains.

The role of the $\mathrm{N}$ terminus in subcellular localization was first investigated for a range of resistance proteins as a prelude to selection of specific proteins for more detailed analysis.

Table 2. Localization of R:GFP and PBS1:GFP fusion proteins in transgenic Arabidopsis plants and bombarded tobacco-leaf epidermal cells ${ }^{\mathrm{a}}$

\begin{tabular}{|c|c|c|c|c|}
\hline \multirow[b]{2}{*}{ Protein } & \multicolumn{2}{|c|}{ Arabidopsis transformant } & \multicolumn{2}{|c|}{$\begin{array}{l}\text { Tobacco leaf epidermal cel } \\
\text { particle bombardment }\end{array}$} \\
\hline & 100N:GFP & 30N:GFP & 100N:GFP & 30N:GFP \\
\hline \multicolumn{5}{|l|}{ Arabidopsis } \\
\hline RPM1 & $\mathrm{CS}^{\mathrm{b}}$ & - & ND & CS \\
\hline RPS2 & $\mathrm{CS} / \mathrm{PM}^{\mathrm{b}}$ & - & ND & $\mathrm{CS} / \mathrm{PM}$ \\
\hline RPS4 & $\mathrm{CS}^{\mathrm{b}}$ & - & ND & $\mathrm{CS}$ \\
\hline RPS5 & PM & PM & PM & PM \\
\hline RPP1-WsA & ER/GA & ER/GA & ER/GA & ER/GA \\
\hline RPP1-WsB & PM & - & - & PM \\
\hline RPP4 & $\mathrm{CS}^{\mathrm{b}}$ & - & ND & $\mathrm{CS}$ \\
\hline RPP8 & $\mathrm{CS}^{\mathrm{b}}$ & - & ND & CS \\
\hline PBS1 & PM & - & PM & - \\
\hline \multicolumn{5}{|l|}{ Flax } \\
\hline L6 & GA & GA & GA & GA \\
\hline M & - & - & $\mathrm{TP}$ & $\mathrm{TP}$ \\
\hline $\mathrm{P} 2$ & - & - & - & CS \\
\hline
\end{tabular}

${ }^{\mathrm{a}} \mathrm{GFP}=$ green fluorescent protein, $100 \mathrm{~N}=$ the $\mathrm{N}$-terminal 100 amino acids, $30 \mathrm{~N}=$ the N-terminal 30 amino acids, $\mathrm{CS}=$ cytosol, $\mathrm{PM}=$ plasma membrane, $\mathrm{ER}=$ endoplasmic reticulum, $\mathrm{GA}=$ Golgi apparatus, $\mathrm{TP}=$ tonoplast, and $\mathrm{ND}=$ localization not determined because only a few abnormal cells showed fluorescence.

${ }^{\mathrm{b}}$ Only weak GFP fluorescence was detected and only in roots.
DNA constructs were made using the Cauliflower mosaic virus (CaMV) $35 \mathrm{~S}$ promoter to express sequences encoding the $\mathrm{N}$ terminal 100 amino acid residues $(100 \mathrm{~N})$ of the Arabidopsis RPM1, RPS2, RPS4, RPS5, RPP1-WsA, RPP1-WsB, RPP4, and RPP8 resistance proteins; the flax L6 resistance protein; and the Arabidopsis PBS1 protein, fused at the $\mathrm{C}$ terminus to GFP. The length of 100 amino acids was chosen arbitrarily to ensure a high probability that all N-terminal targeting information would be included in the fusion proteins. The $100 \mathrm{~N}$ fusions then provided a benchmark for subsequent shorter fusions for which there was a greater risk that $\mathrm{N}$-terminal targeting information could have been lost. Transgenic Arabidopsis plants expressing these constructs were generated and fusion protein localization was examined in leaf and root tissues by confocal laser-scanning fluorescence microscopy (Fig. 1A). The RPM1-
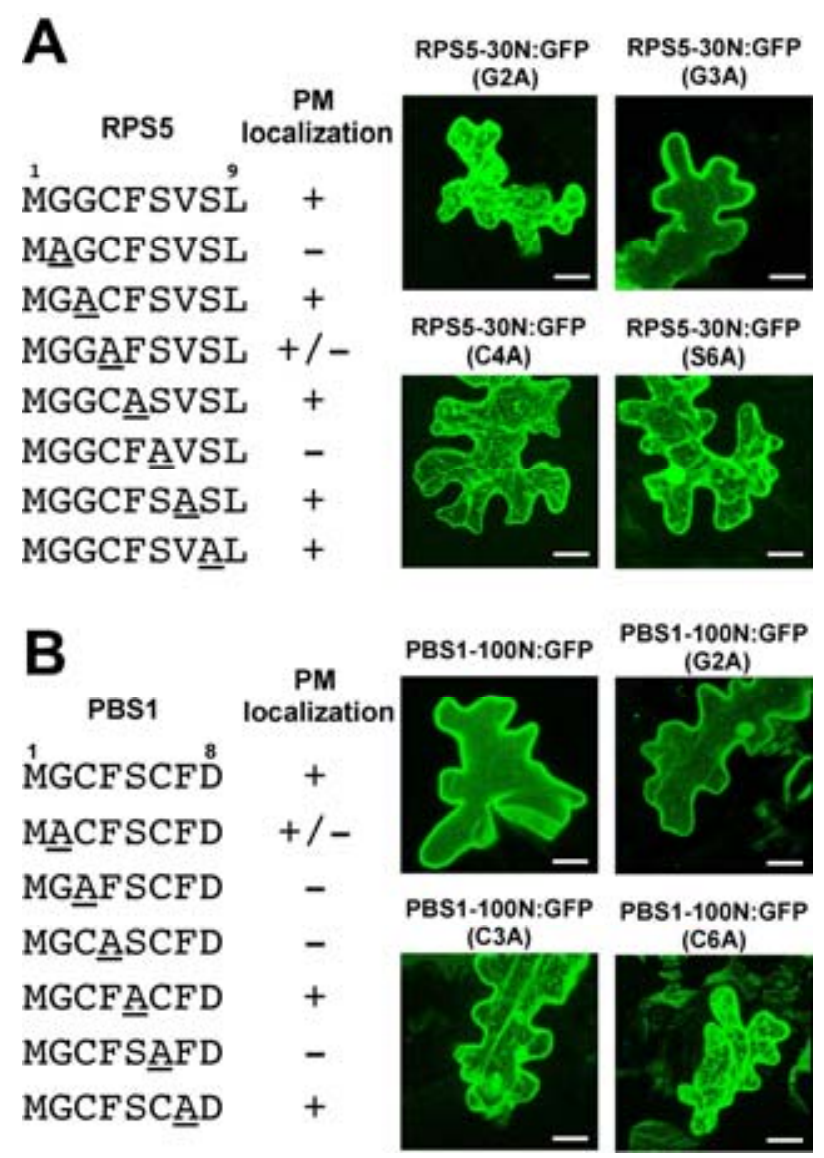

Fig. 2. Mutational analysis of N-terminal residues required for plasma membrane localization of the fusion proteins containing $\mathbf{A}$, the $\mathrm{N}$-terminal 30 amino acids $(30 \mathrm{~N})$ of RPS5 or B, 100 amino acids $(100 \mathrm{~N})$ of PBS1 fused to green fluorescent protein (GFP). A, Summary and examples of localization patterns observed in epidermal cells of bombarded tobacco leaves for alanine-scanning mutants of the subterminal seven amino acids of RPS5 in fusion proteins containing the $30 \mathrm{~N}$ of RPS5 fused to GFP. The wild-type progenitor and the G3A mutant show plasma membrane localization whereas the G2A and S6A mutants show strong nucleo-cytosolic localization. The C4A mutation shows less nucleo-cytosolic labeling than the G2A and S6A mutants, consistent with a smaller shift from plasma membrane localization to nucleo-cytosolic localization. Bars $=20 \mu \mathrm{m}$. B, Summary and examples of localization patterns observed in epidermal cells of bombarded tobacco leaves for alanine-scanning mutants of the subterminal six amino acids of PBS1 in fusion proteins containing the $100 \mathrm{~N}$ of PBS1 fused to GFP. The wild-type progenitor shows plasma membrane localization whereas the $\mathrm{C} 3 \mathrm{~A}$ and $\mathrm{C} 6 \mathrm{~A}$ mutants both show nucleo-cytosolic localization. The G2A mutant shows less nucleo-cytosolic labeling than the $\mathrm{C} 3 \mathrm{~A}$ and $\mathrm{C} 6 \mathrm{~A}$ mutants, consistent with a smaller shift from plasma membrane localization to nucleo-cytosolic localization. Bars = $20 \mu \mathrm{m}$. 
100N:GFP, RPS4-100N:GFP, RPP4-100N:GFP, and RPP8100N:GFP fusions showed nucleo-cytosolic fluorescence (Fig. 1A; Supplementary Fig. S1), suggesting that these proteins do not contain any specific N-terminal targeting sequences (note that the molecular masses of these GFP fusion proteins fall below the nuclear exclusion limit so that they are able to diffuse freely into the nucleus). However, the RPS5-100N:GFP, RPP1WsB-100N:GFP, and PBS1-100N:GFP fusions showed a pattern of GFP fluorescence consistent with PM localization (Fig. 1A). The RPS2-100N:GFP fusion showed a pattern of GFP fluorescence indicative of both PM and nucleo-cytosolic localization (data not shown) (similar to the $30 \mathrm{~N}$ fusion described below [Fig. 1B]). In contrast, the RPP1-WsA-100N:GFP fusion showed a reticulate pattern of fluorescence indicative of ER as well as large elongated spots of fluorescence consistent with ER bodies, a characteristic of Arabidopsis (Gunning 1998), and small bright spots of fluorescence that may correspond to the GA superimposed on the reticular network (Fig. 1A). The L6-100N:GFP fusion showed a punctate pattern of fluorescence consistent with localization to the GA (Fig. 1A). These data are summarized in Table 2.

DNA constructs were also made using the CaMV 35S promoter to express sequences encoding the N-terminal 30 amino acid residues (30N) of the Arabidopsis RPM1, RPS2, RPS4, RPS5, RPP1-WsA, RPP1-WsB, RPP4, and RPP8 resistance proteins and the flax L6, M, and P2 resistance proteins, fused at the $\mathrm{C}$ terminus to the $\mathrm{N}$ terminus of GFP. The RPS530N:GFP, RPP1-WsA-30N:GFP, and L6-30N:GFP constructs, were used to produce transgenic Arabidopsis plants. These showed the same patterns of localization as the corresponding $100 \mathrm{~N}$ fusions (Table 2), indicating that the membrane targeting information of these proteins was contained in the first 30 amino acids.

100N:GFP and 30N:GFP fusions were also examined in bombarded epidermal cells of tobacco leaves and showed the same localization as observed in transgenic Arabidopsis plants (Fig. 1B; Table 2). 100N:GFP and 30N:GFP fusions of the flax $\mathrm{M}$ resistance protein showed globular vacuolar invaginations (Saito et al. 2002) and transvacuolar strands indicative of tono- plast (TP) localization (Fig. 1B; Table 2; Supplementary Fig. $\mathrm{S} 2$ ), although close apposition of the TP to the PM in epidermal cells did not exclude additional localization to the PM. A 30N:GFP fusion of the flax P2 resistance protein, which has no predicted targeting sequences, showed a pattern of GFP fluorescence consistent with nucleo-cytosolic localization (Fig. 1B; Table 2; Supplementary Fig. S3).

\section{Mutations of the predicted myristoylation or palmitoylation motifs of RPS5 and PBS1 prevent membrane attachment.}

The $\mathrm{N}$ terminus of RPS5 contains a motif (MGxxxS/T) predicted to direct myristoylation of the subterminal glycine residue (Johnson et al. 1994), while PBS1 has a related sequence with cysteine in place of the conserved S/T (Fig. 2). Both also contain nearby cysteine residues that could act as sites for palmitoylation. Alanine-scanning mutagenesis of these motifs in the RPS5-30N:GFP and PBS1-100N:GFP fusions was used to test the role of these regions in membrane attachment (Fig. 2). The G3A mutation did not affect PM localization of the RPS5-30N:GFP fusion but the G2A and S6A mutations showed strong nucleo-cytosolic fluorescence (Fig. 2A), indicating a major loss in the ability of the fusion protein to attach to the $\mathrm{PM}$, consistent with the roles of $\mathrm{G} 2$ and S6 in myristoylation. The C4A mutation showed weaker nucleo-cytosolic fluorescence than the G2A or S6A mutations, indicating a less severe reduction in the ability of the RPS5-30N:GFP fusion protein to attach to the PM. These results are consistent with the wellestablished primary role of the subterminal glycine as a substrate for myristoylation (Johnson et al. 1994), with the resultant membrane association, in turn, promoting palmitoylation of nearby cysteines, thereby stabilizing membrane attachment (Shahinian and Silvius 1995). Interestingly, the relative importance of the glycine and cysteine residues was reversed in the PBS1-100N:GFP fusion, with the C3A and C6A mutations both showing strong nucleo-cytosolic fluorescence but the G2A mutation showing weaker nucleo-cytosolic fluorescence (Fig. 2B). Surprisingly, despite no predicted role for F4 in myristoylation or palmitoylation, the F4A mutation also showed
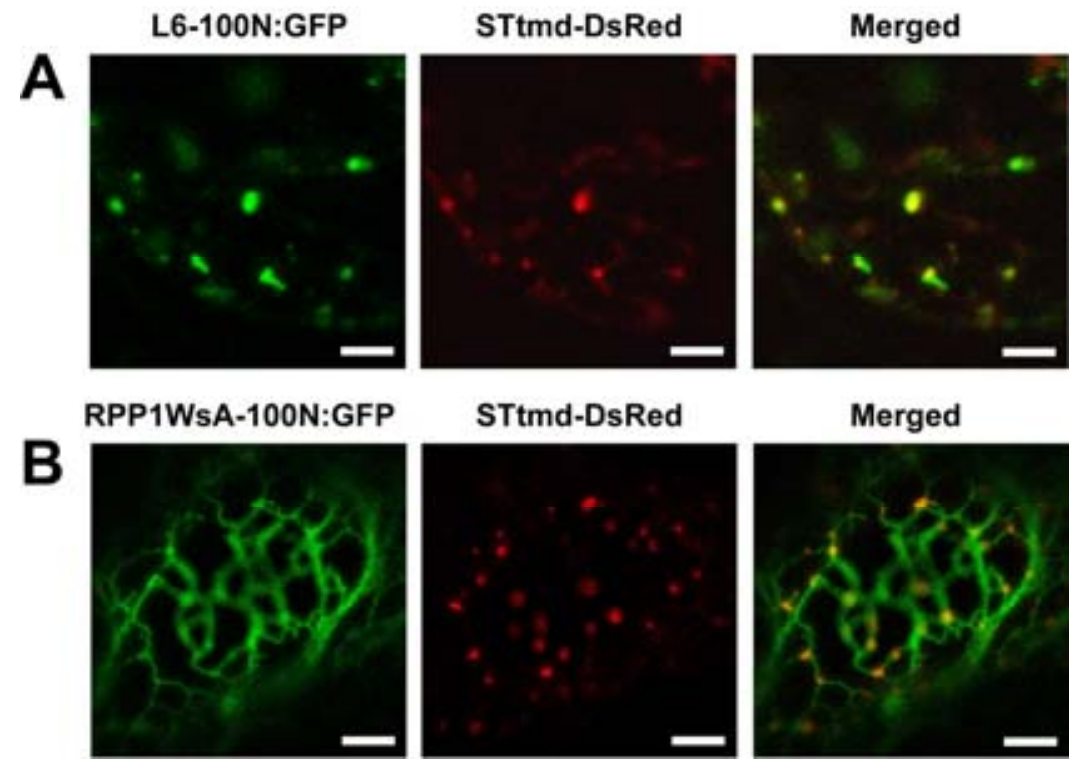

Fig. 3. Co-localization of L6-N-terminal 100 amino acid (100N):green fluorescent protein (GFP) and RPP1-WsA-100N:GFP fusion proteins with an STtmd:DsRed fusion protein marking the Golgi apparatus. A, High magnification view of part of a tobacco leaf epidermal cell co-bombarded with L6100N:GFP and STtmd:DsRed fusion proteins showing GFP fluorescence in the left panel, DsRed fluorescence in the central panel, and the merged images in the right panel. Bars $=10 \mu \mathrm{m}$. B, High-magnification view of part of a tobacco leaf epidermal cell co-bombarded with RPP1-WsA-100N:GFP and STtmd:DsRed fusion proteins showing GFP fluorescence in the left panel, DsRed fluorescence in the central panel, and the merged images in the right panel. Bars $=10 \mu \mathrm{m}$. 
strong nucleo-cytosolic fluorescence (Fig. 2B) indicating a major loss in the ability of the PBS1-100N:GFP fusion protein to attach to the PM.

\section{L6 and RPP1-WsA signal anchors localize GFP to the cytosolic face of the GA.}

In Arabidopsis transformants and bombarded tobacco leaf epidermal cells, the L6:GFP fusions gave a punctate pattern of fluorescence consistent with GA localization and the RPP1WsA fusions gave a pattern of fluorescence that could be consistent with labeling of the GA and ER (Fig. 1A). To test for GA localization, the L6-100N:GFP and RRP1-WsA100N:GFP fusions were each co-bombarded with a plasmid encoding the GA marker STtmd (rat sialyl transferase transmembrane domain) (Boevink et al. 1998) fused to DsRed. The STtmd:DsRed fusion shows punctate labeling that co-localizes with the L6-100N:GFP and RPP1-WsA-100N:GFP fusions (Fig. 3), indicating that the L6-100N:GFP fusion localizes to the GA and the RPP1-WsA-100N:GFP fusion localizes to the GA as well as the ER. Interestingly, whereas there is no red fluorescence corresponding to STtmd:DsRed alone in the merged image shown in Figure 3A, there is green fluorescence corresponding to L6-100N:GFP alone. This could be attributed, in part, to the GFP fluorescence being brighter than the DsRed fluorescence. However, it should be noted that the areas of green fluorescence alone are always contiguous with the areas of overlapping red and green fluorescence; therefore, it is possible that L6 may be distributed a little more broadly in the GA than ST, which marks the trans Golgi.

Localization of L6:GFP fusions to the GA and RPP1WsA:GFP fusions to the ER and GA does not enable discrimination between location of the proteins in the lumen of the ER or GA, insertion into the luminal face of the ER or GA membrane (type II signal anchor), or, as expected, insertion into the cytosolic face of the ER or GA membrane (type I signal anchor). A type I signal-anchored protein has its $\mathrm{C}$ terminus exposed to the cytosol whereas a type II signal-anchored protein has its $\mathrm{N}$ terminus exposed to the cytosol. To examine the topology of L6 and RPP1-WsA, the AvrRpt 2 cysteine protease cleavage site from RIN4 (RIN4cs) was engineered between the signal anchor and GFP sequences. The L6-100N:RIN4cs:GFP and RPP1-WsA-100N:RIN4cs:GFP fusions were bombarded into tobacco leaves together with a construct expressing AvrRpt2 or an empty vector. In the absence of AvrRpt2, fusions carrying RIN4cs gave the same pattern of localization as fusions lacking RIN4cs but, when co-bombarded with AvrRpt2, the RIN4cs fusions showed nucleo-cytosolic fluorescence consistent with release of GFP from the cytosolic face of the ER or GA membranes (Fig. 4). Co-expression of the unmodified L6100N:GFP and RPP1-WsA-100N:GFP fusions with AvrRpt2 did not affect their localization. These data show that the $\mathrm{N}$ terminal domains of L6 and RPP1-WsA contain type I signal anchors that are able to attach L6 to the GA membrane and RPP1-WsA to the ER and GA membranes, leaving the bulk of the protein in the cytosol.

\section{The M signal anchor localizes to the TP.}

To further resolve whether the M:GFP fusion proteins localize exclusively to the TP or also to the PM, the M-30N:GFP fusion was expressed in tobacco epidermal cells by Agrobacterium-mediated gene transfer following infiltration (agroinfiltration) of tobacco leaves. Close examination of epidermal cells in these leaves revealed that membrane labeling occurred on the cytosolic side but not the PM side of nuclei and chloroplasts appressed to the PM (Fig. 5A), indicating that the PM is not labeled. In addition, plasmolysis of cells expressing $\mathrm{M}$ $30 \mathrm{~N}$ :GFP resulted in the appearance of multiple flask-like in- vaginations of the labeled membrane (Fig. 5B), consistent with osmotic stress-induced folding of the TP as described by Reisen and associates (2005). This response to plasmolysis was quite distinct from that observed for the PM-localized PBS1-100N: GFP fusion protein, which showed lunate labeling consistent with retraction of the PM from the cell wall except at points of cell wall attachment (Fig. 5C).

\section{Localization of full-length L6 protein.}

GFP fusions to the N-terminal domains of resistance proteins can reveal localization signals contained within these domains but do not necessarily reflect the localization of the fulllength resistance proteins. To examine the relationship between the $\mathrm{N}$-terminal localization signals and resistance protein localization, a fusion was constructed between the citrine (CIT) variant of GFP (Griesbeck et al. 2001) and full-length L6. The L6:CIT fusion was placed under the control of L6 regulatory sequences and used to generate stable flax transformants. Leaf epidermal cells of flax plants expressing the L6:CIT

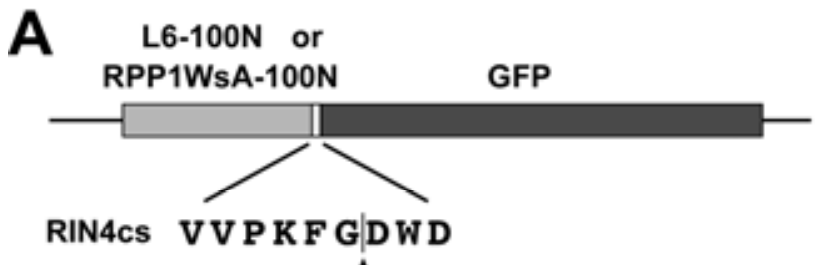

B
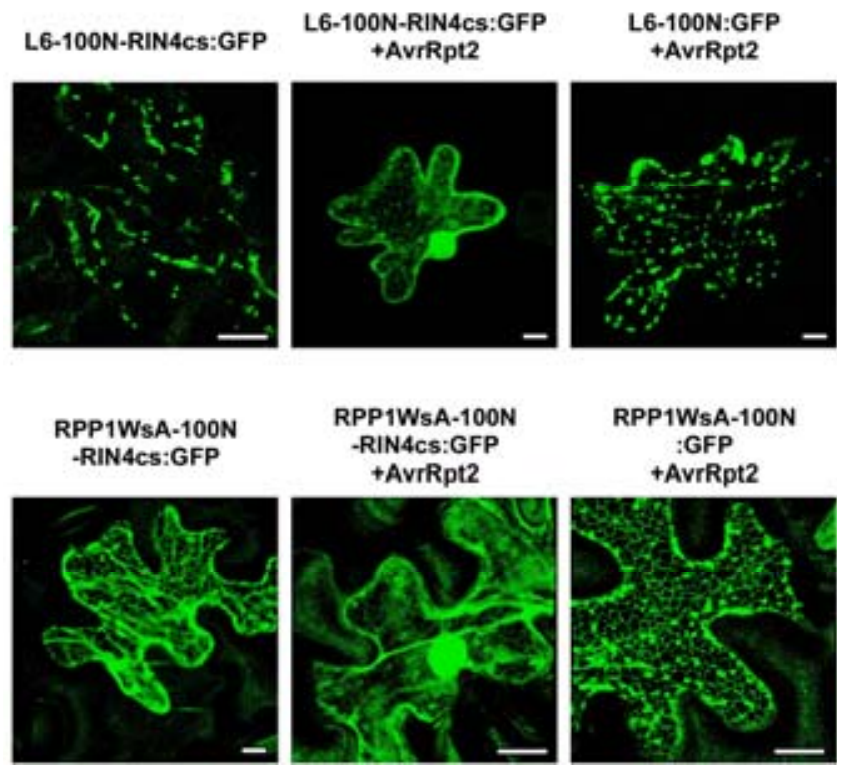

Fig. 4. L6-N-terminal 100 amino acid (100N):green fluorescent protein (GFP) and RPP1-WsA-100N:GFP fusion proteins are inserted into the cytosolic face of the membrane. A, Diagram of the L6-100N:GFP and RPP1-WsA-100N:GFP fusion constructs modified to include a nine amino acid sequence derived from RIN4 containing an AvrRpt 2 cleavage site (RIN4cs). The site of cleavage by AvrRpt2 is arrowed. B, Localization patterns observed in epidermal cells of bombarded tobacco leaves for the L6100N:RIN4cs:GFP and RPP1-WsA-100N:RIN4cs:GFP fusion proteins on their own (left panels) or co-expressed with AvrRpt2 (middle panels), and the L6-100N:GFP and RPP1-WsA-100N:GFP fusion proteins (i.e., lacking the AvrRpt2 cleavage site) co-expressed with AvrRpt2 (right panels). Left panels show that the localization patterns of the fusion proteins have not been altered by the introduction of the AvrRpt 2 cleavage site. Middle panels show the release of GFP into the cytosol following cleavage by AvrRpt2. Right panels show that the release of GFP by AvrRpt2 is dependent on the presence of the AvrRpt2 cleavage site. Bars $=10 \mu \mathrm{m}$. 
fusion showed the same patterns of fluorescence as the L630N:GFP fusion (Fig. 6A), consistent with GA localization of L6. Moreover, treatment of flax leaves expressing L6:CIT with brefeldin A, which disrupts the integrity of Golgi stacks in plants (Ritzenthaler et al. 2002), resulted in the formation of brefeldin A-induced aggregates (Fig. 6B), confirming GA localization. Transgenic flax plants expressing the L6:CIT fusion showed a necrotic response to agroinfiltration with AvrL567
(Fig. 6C), and AvrL567-dependent resistance to flax rust, indicating that L6 function was not affected by CIT.

\section{Mutational analysis}

of the $L 6$ and $M$ signal anchor domains.

GA localization of L6-30N:GFP and TP localization of M$30 \mathrm{~N}$ :GFP in bombarded tobacco leaf epidermal cells indicates that membrane-specific attachment information is con-
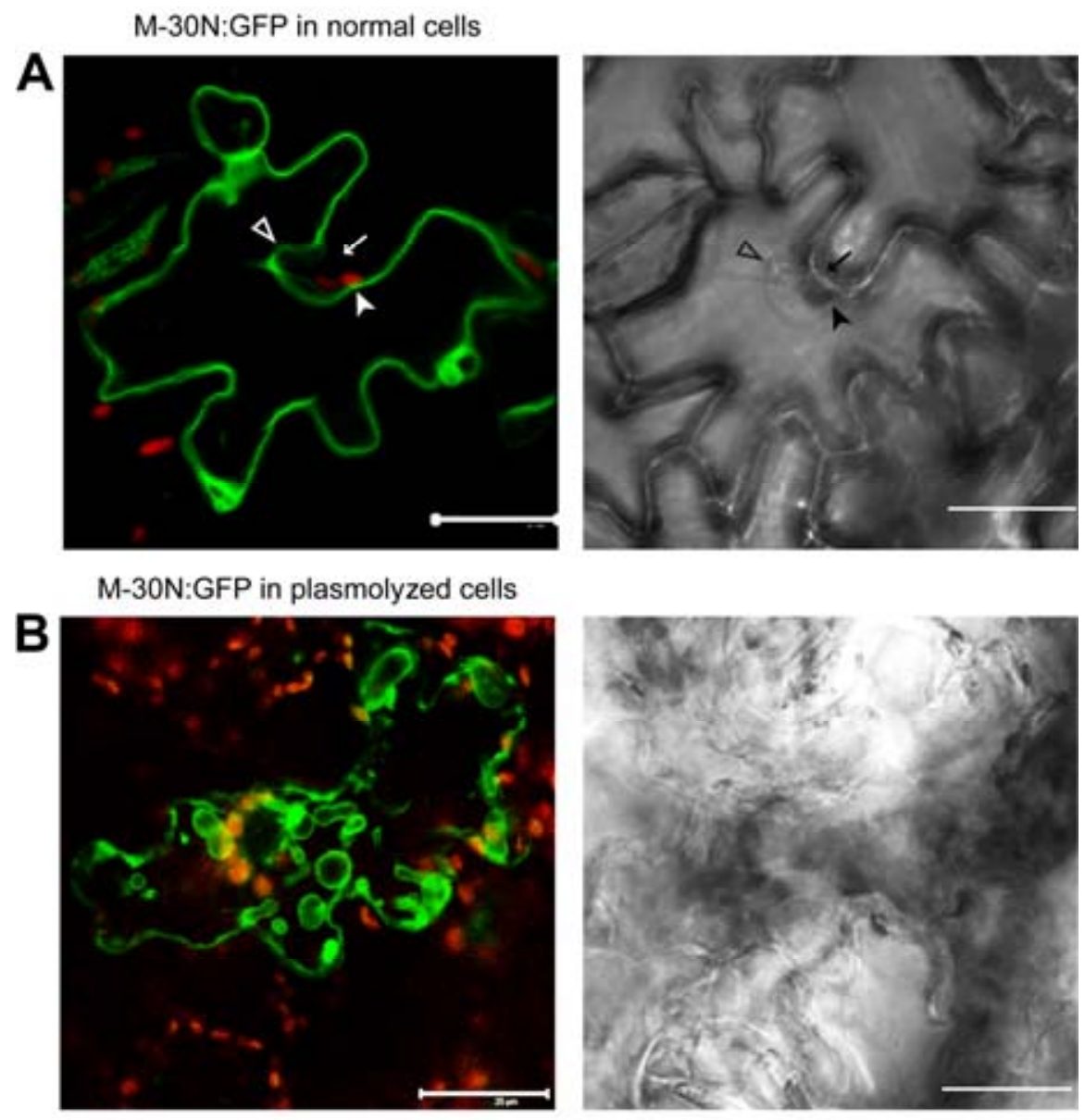

PBS1-100N:GFP in plasmolyzed cells
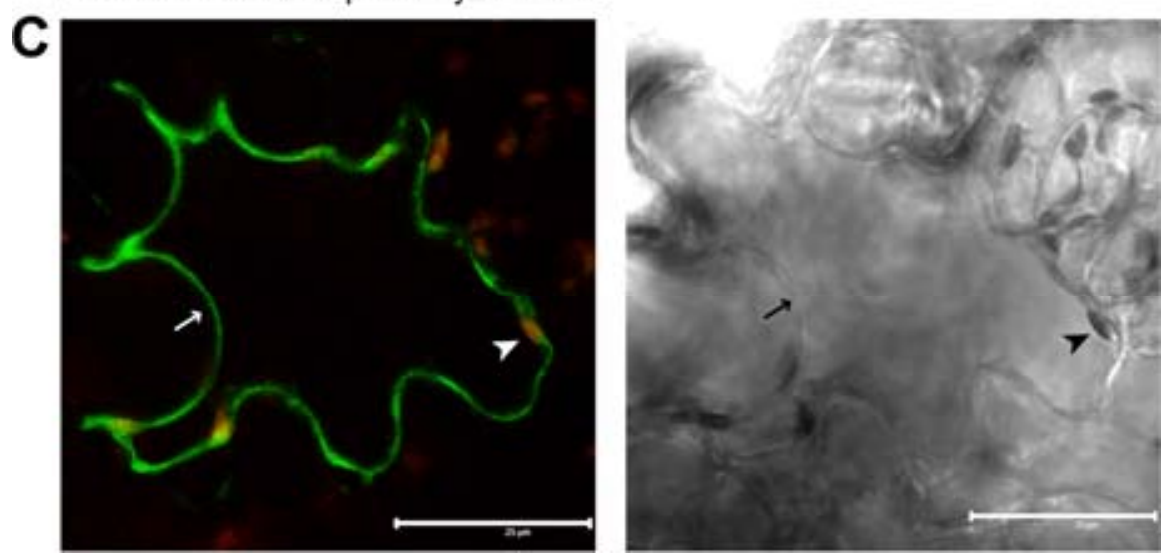

Fig. 5. M-N-terminal 30 amino acid (30N):green fluorescent protein (GFP) fusion proteins localize to the tonoplast but not the plasma membrane. A, M$30 \mathrm{~N}$ :GFP fusion proteins show localization to tonoplast membrane (internal to the nucleus and chloroplasts) but not to plasma membrane (external to the nucleus and chloroplasts) in epidermal cells of agroinfiltrated tobacco leaves. Bars $=25 \mu \mathrm{m}$. B, M-30N:GFP fusion protein shows tonoplast localization characterized by a convex pattern of membrane labeling and a marked increase in the number of vacuolar invaginations (circular or flask-shaped in cross section) in plasmolyzed cells. Bars $=25 \mu \mathrm{m}$. C, PBS1-N-terminal 100 amino acid $(100 \mathrm{~N})$ :GFP fusion protein shows plasma membrane localization characterized by concave patterns of membrane labeling flanked by points of membrane attachment to the cell wall in plasmolyzed cells. Bars $=25 \mu \mathrm{m}$. Leaves in B and C were plasmolyzed by infiltrating with $0.8 \mathrm{M}$ mannitol just prior to imaging. Left panels in A, B, and C show green fluorescence marking the location of the GFP fusion proteins and red chlorophyll fluorescence marking the location of chloroplasts. Right panels show the corresponding bright-field image showing the outline of the cell and the position of the nucleus (open arrowheads), the chloroplasts (solid arrowheads), and the plasma membrane (arrows). 
tained within the first 30 amino acids of L6 and M. The N termini of L6 and $\mathrm{M}$ are identical for the first 15 amino acids, apart from a conservative E6D substitution (Fig. 7A). This suggests that residues determining GA versus TP localization are located downstream of the first 15 amino acid residues. To further test this hypothesis, a mutant of the L6-30N:GFP construct was made in which residues 6 to 15 of L6 (preceding a helix-breaking proline residue) were replaced by residues 5 to 14 of GA- and ER-localized RPP1-WsA (preceding a helix-breaking glycine residue). Tobacco leaf cells bombarded with this construct showed a pattern of GFP fluorescence consistent with GA localization only (Supplementary Fig. S4), confirming that no membrane-specific localization information is contained in residues 5 to 14 of RPP1WsA or 6 to 15 of L6.

To investigate the role of residues downstream of the signal anchor in membrane localization, we introduced $M$ residues into the predicted signal anchor domain of L6-30N:GFP (residues 7 to 24) and L6 residues or alanine residues into corresponding positions in the $\mathrm{N}$ terminus of $\mathrm{M}-30 \mathrm{~N}$ :GFP (Fig. 7). Introduction of P16D and F17N substitutions in L6$30 \mathrm{~N}: \mathrm{GFP}(\mathrm{m} 1)$ resulted in a shift in GFP localization from the GA to the ER, whereas the L19C, L20C, and N21G substitutions in L6-30N:GFP(m2) caused a shift in fluorescence from the GA toward the TP (Fig. 7). L6-30N(m3):GFP, which combines all five substitutions, localized almost exclusively to the TP. M-30N(m1):GFP carrying C19A, C20A, and G21A mutations showed a shift from TP localization toward the cytosol whereas M-30N(m2):GFP encoding D16P and N17F substitutions in addition to the C19A, C20A, and G21A substitutions showed a shift in localization from the TP to the GA (Fig. 7). In total, these data suggest that membrane-specific localization information is contained in residues 16 to 21 of L6 and M.
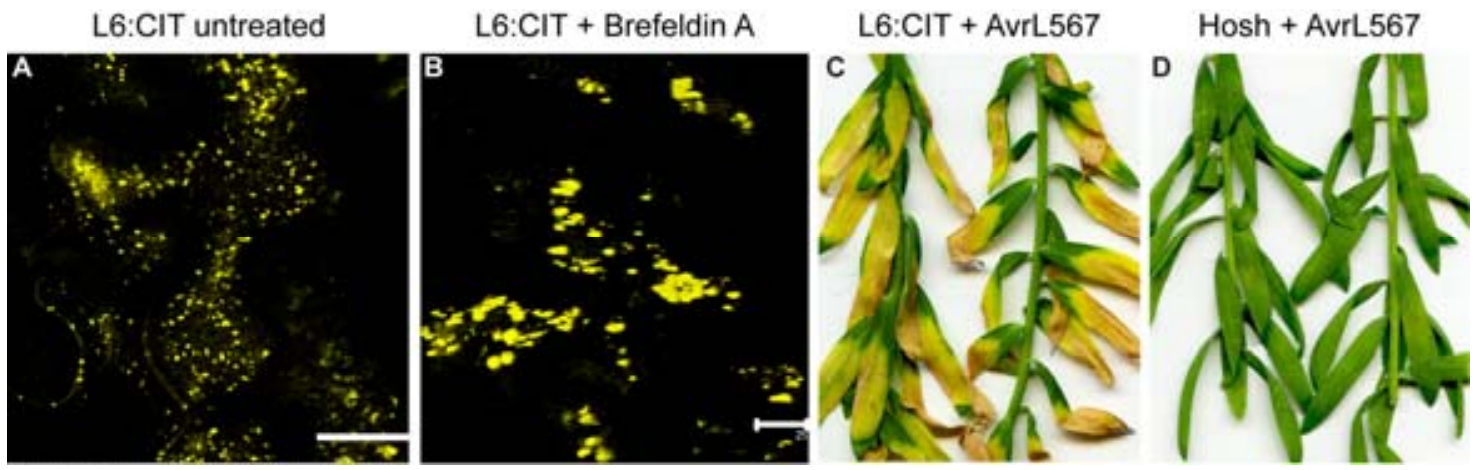

Fig. 6. Full-length L6:citrine (CIT) fusion protein localizes to the Golgi apparatus. A, Full-length flax L6 resistance protein fused to the CIT variant of green fluorescent protein (GFP) shows localization to the Golgi apparatus in epidermal cells of transgenic flax plants. Bar $=25 \mu \mathrm{m}$. B, Treatment with brefeldin A alters the pattern of CIT fluorescence in epidermal cells of transgenic flax plants consistent with localization of the L6:CIT to the Golgi apparatus. Leaves were infiltrated with $50 \mu \mathrm{M}$ brefeldin A 30 min prior to imaging. Bar $=25 \mu \mathrm{m}$. Agrobacterium spp.-mediated transient expression of AvrL567dSP (lacking the signal peptide) induces chlorosis and necrosis in $\mathbf{C}$, leaves of transgenic flax plants expressing L6:CIT but not in D, untransformed Hoshangabad (Hosh) flax plants. Plants were photographed 11 days after infiltration.

A

$\begin{array}{llll}\text { L6-30N } & \text { MSYLREVATAVALLLPFILLNKFWRPNSKD } & \text { GA } \\ \text { L6-30N(m1) } & \text { MSYLREVATAVALLLDNILLNKFWRPNSKD } & \text { GAER } \\ \text { L6-30N(m2) } & \text { MSYLREVATAVALLLPFICCGKFWRPNSKD } & \text { TP/GACS } \\ \text { L6-30N(m3) } & \text { MSYLREVATAVALLLDNICCGKFWRPNSKD } & \text { TP } \\ \text { M-30N } & \text { MSYLRDVATAVALLLDNLCCGRPNLNNDNE } & \text { TP }\end{array}$

B

$M-30 N$
$M-30 N(m 1)$
$M-30 N(m 2)$
$L 6-30 N$

\section{MSYLRDVATAVALLLDNLCCGRPNLNNDNE TP MSYLRDVATAVALLLDNLAAARPNLNNDNE MSYLRDVATAVALLLPFLAAARPNLNNDNE MSYLREVATAVALLLPFILLNKFWRPNSKD}

C

L6-30N(m1):GFP

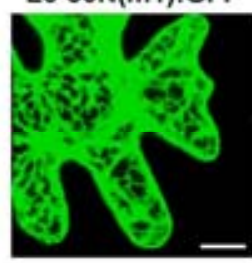

M-30N(m1):GFP

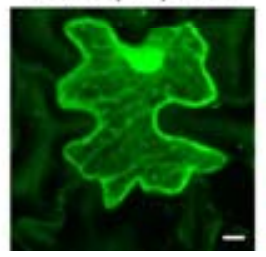

L6-30N(m3):GFP

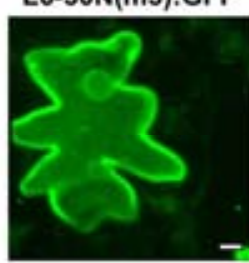

M-30N(m2):GFP

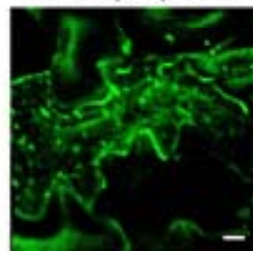

Fig. 7. Specific amino acid substitutions adjacent to the signal anchor domain can alter the pattern of localization of L6 to that of M and vice versa. A, Introduction of specific amino acids from the N-terminal sequence of M into the N-terminal sequence of L6 disrupts localization of the L6-N-terminal 30 amino acid $(30 \mathrm{~N})$ : green fluorescent protein (GFP) fusion protein to the Golgi apparatus and leads to tonoplast localization in bombarded tobacco leaf epidermal cells. An N-terminal sequence alignment is shown with the progenitor L6 sequence at the top and the M sequence at the bottom, with the mutant L6 sequences shown in between. The predicted transmembrane domain of L6 is underlined. The localization pattern observed for each sequence is shown to the right of the alignment. $\mathrm{CS}=$ cytosol, $\mathrm{ER}=$ endoplasmic reticulum, $\mathrm{GA}=$ Golgi apparatus, and TP = tonoplast. B, Introduction of alanines or specific amino acids from the N-terminal sequence of L6 into the N-terminal sequence of M disrupts localization of the M-30N:GFP fusion protein to the tonoplast and leads to localization in the Golgi apparatus in bombarded tobacco leaf epidermal cells. An N-terminal sequence alignment is shown with the progenitor $\mathrm{M}$ sequence at the top and the L6 sequence at the bottom, with the mutant M sequences shown in between. The predicted transmembrane domain of L6 is underlined. The localization pattern observed for each sequence is shown to the right of the alignment. C, Examples of localization patterns observed for the mutants in A and B. Bars $=10 \mu \mathrm{m}$. 


\section{Palmitoylation is required for membrane attachment of PBS1 but not $M$.}

To examine the possible palmitoylation of the N-terminal membrane-attachment domain of the $M$ resistance protein, tobacco leaves were agroinfiltrated with M-30N:GFP or PBS1100N:GFP. Then, 1 and 2 days later, the same leaves were infiltrated with the palmitoylation inhibitor 2-bromopalmitic acid (2-BPA) or the solvent (1\% dimethyl sulfoxide) alone as described by Kim and associates (2005). The patterns of GFP fluorescence were examined 3 days after agroinfiltration.
Leaves treated with PBS1-100N:GFP and $100 \mu \mathrm{M}$ 2-BPA showed a clear shift toward a nucleo-cytosolic pattern of fluorescence (Fig. 8A) whereas the solvent-treated control showed an unaltered pattern of PM labeling. Leaves treated with M-30N:GFP and $100 \mu \mathrm{M}$ 2-BPA showed no shift toward a nucleo-cytosolic pattern of fluorescence (Fig. 8B). Plasmolysis of leaves treated with M-30N:GFP and $100 \mu \mathrm{M} 2-\mathrm{BPA}$ again showed flask-like invaginations of the TP membrane and revealed no evidence for GFP fluorescence in the cell wall or the PM (Fig. 8C).
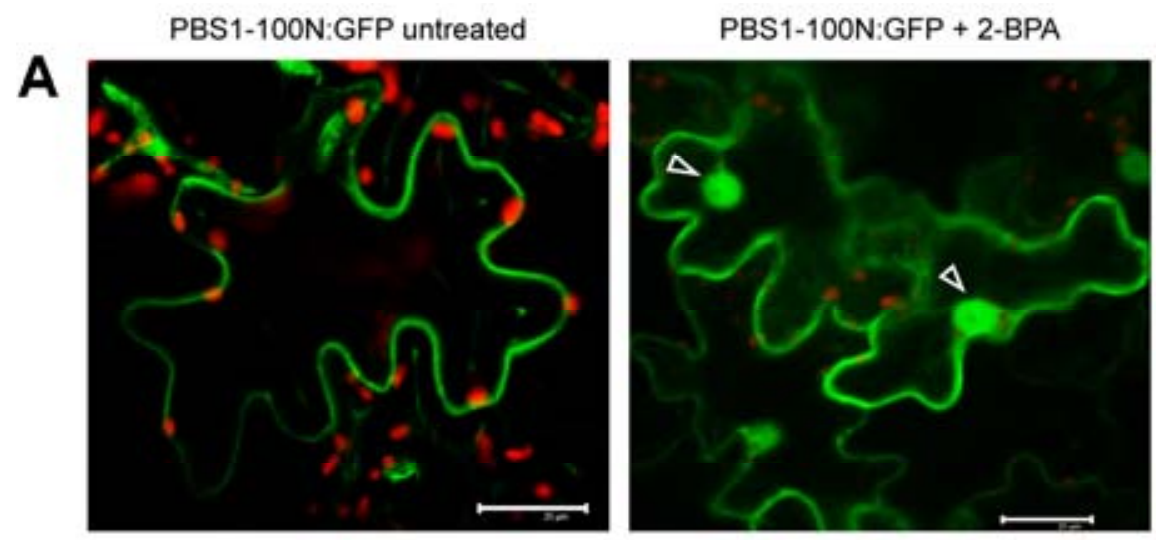

M-30N:GFP+2-BPA in normal cells
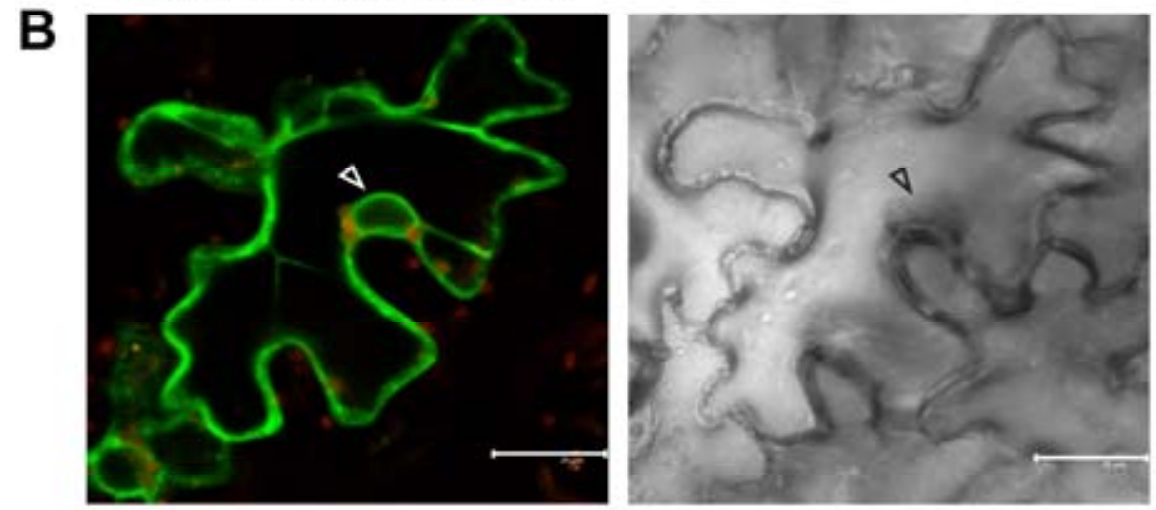

\section{M-30N:GFP+2-BPA in plasmolysed cells}
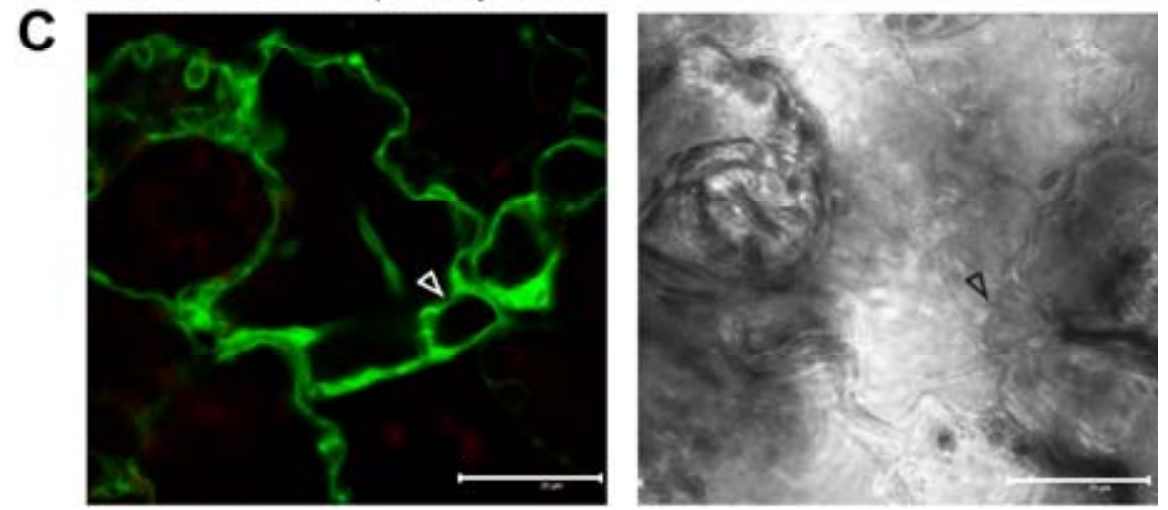

Fig. 8. Treatment with the palmitoylation inhibitor 2-bromopalmitic acid (2-BPA) causes a shift toward nucleo-cytosolic localization for the PBS1-N-terminal 100 amino acid $(100 \mathrm{~N})$ : green fluorescent protein (GFP) fusion protein but not the M-N-terminal 30 amino acid (100N):GFP fusion protein. A, Epidermal cells of tobacco leaves agroinfiltrated with PBS1-100N:GFP and treated with $100 \mu \mathrm{M}$ 2-BPA (right panel) show nuclear fluorescence (open arrowheads), indicating a shift toward nucleo-cytosolic localization, whereas untreated cells show no nuclear fluorescence (left panel). Note that the molecular mass of the GFP fusion protein falls below the nuclear exclusion limit for soluble proteins and, therefore, the protein can diffuse freely from the cytosol into the nucleus. B, Epidermal cells of tobacco leaves agroinfiltrated with M-30N:GFP and treated with $100 \mu \mathrm{M}$ 2-BPA. C, Epidermal cells of tobacco leaves agroinfiltrated with M-30N:GFP, treated with $100 \mu \mathrm{M}$ 2-BPA, and plasmolyzed by infiltrating with $0.8 \mathrm{M}$ mannitol just prior to imaging. Color panels in $\mathrm{A}, \mathrm{B}$, and $\mathrm{C}$ show green fluorescence marking the location of the GFP fusion proteins and red chlorophyll fluorescence marking the location of chloroplasts. Left panels in B and C show no fluorescence inside the nucleus (open arrowhead) and the localization of M-30N:GFP remains associated with the tonoplast. Right panels in B and C show the corresponding bright-field images showing the outline of the cell and the position of the nucleus (open arrowhead). Bars $=25 \mu \mathrm{m}$. 
N-terminal domain swaps between L6, M, and

P2 resistance proteins show that the membrane attachment domain of L6 contributes to resistance against flax rust.

To investigate the functional significance of membrane attachment in L6 resistance, domain swaps were constructed in which the N-terminal sequences of L6 upstream of the conserved TIR domain were replaced by the corresponding sequences of $\mathrm{M}$ and P2 (Fig. 9A). The M-L6 domain swap, containing the $\mathrm{N}$ terminal membrane-attachment domain from $\mathrm{M}$, and the P2L6 domain swap, containing no N-terminal membrane-attachment domain, were used to generate transgenic flax plants whose progeny were tested for resistance to a strain of flax rust expressing AvrL567. The M-L6 domain swap conferred resistance equivalent to that of L6 in six of seven independent transformants (the seventh was fully susceptible), with pinprick hypersensitive flecks and no rust growth. Transgenic plants expressing the P2-L6 domain swap exhibited partial resistance in 8 of 12 independent transformants, with large necrotic spots on lower leaves and some pustule development on upper leaves (Fig. 9B). Two of the remainder were susceptible and two were fully resistant. These data suggest that the P2-L6 domain swap shows a lower level of resistance than L6.

The subcellular localization of these full-length domain-swap proteins was examined by confocal fluorescence microscopy of epidermal cells in Nicotiana benthamiana leaves agroinfiltrated with the corresponding CIT fusions expressed under the control of the L6 promoter. The full-length L6:CIT fusion showed GA localization (Fig. 10A), as in transgenic flax (Fig. 6A), and the full-length M-L6:CIT fusion showed TP localization (Fig. 10B), consistent with previous observations using L6-30N:GFP and M-30N:GFP fusions; however, the P2-L6:CIT fusion showed no detectable fluorescence. However, when expressed under the control of the CaMV 35S promoter, a full-length P2-L6:YFPv (the Venus variant of yellow fluorescent protein [Nagai et al. 2002]) fusion showed cytosolic localization (Fig. 10C). This observation suggested the P2-L6:CIT fusion may not have accu-

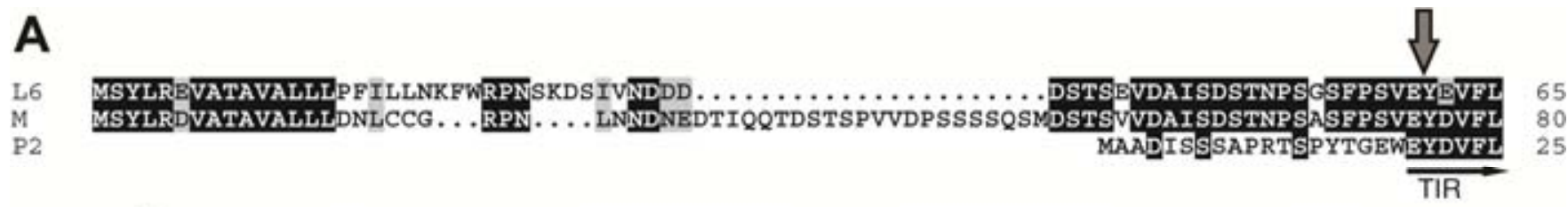

B

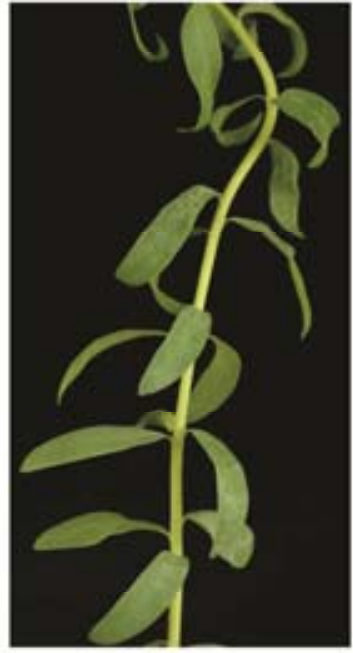

Upper leaf

Lower leaf

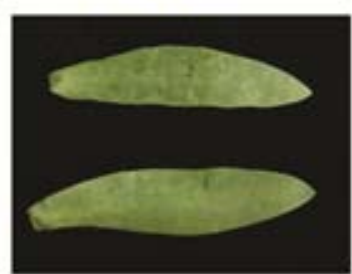

P2-L6 (\#3293)
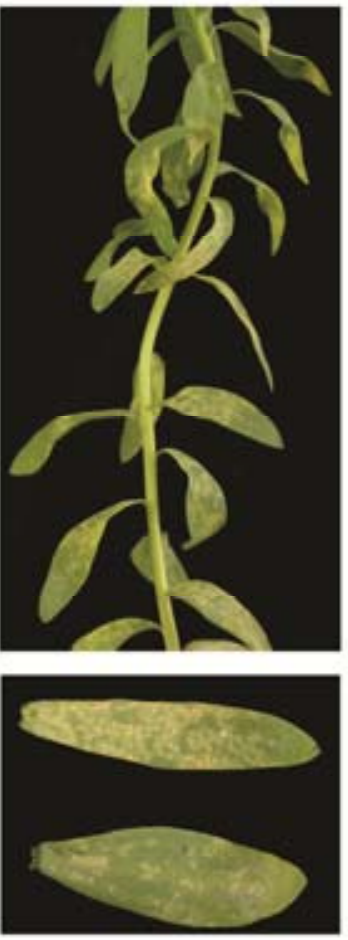

P2-L6 (\#3321)
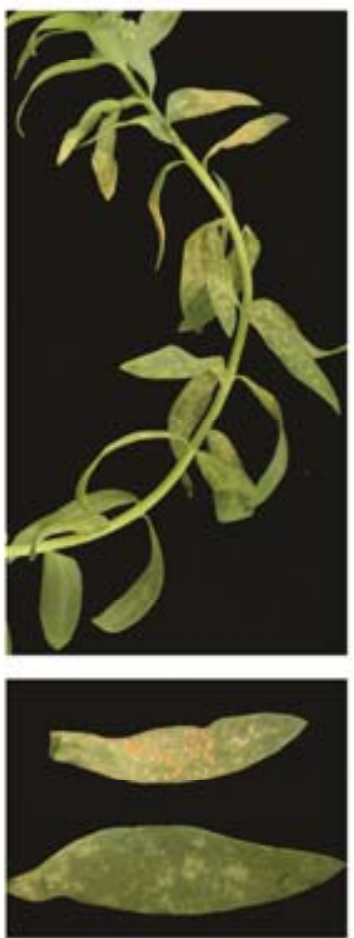

Ward
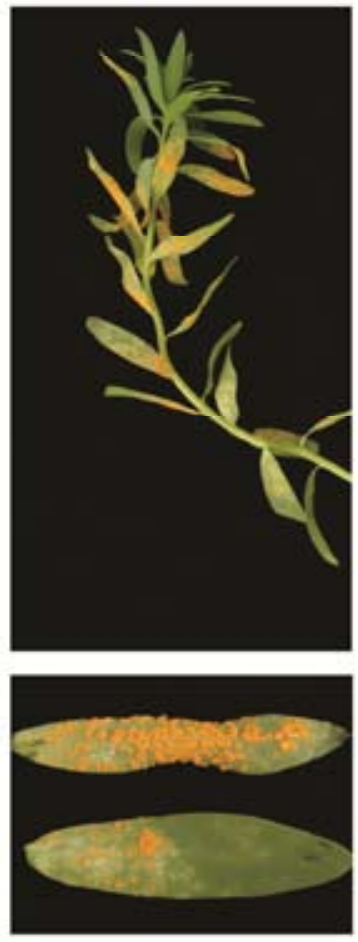

C

L-L11 MSYLREVATAVALLLPFILLVIKFWRPNSKDSIVNDDD $\ldots \ldots \ldots \ldots \ldots \ldots \ldots \ldots \ldots$ DSTSEVDAISDSTMPSGSFPSVEYEVFL 65 M1, M3 MSYLRDVATAVALLLPFILLYKFWRRNSKHSIVNDDD $\ldots \ldots \ldots \ldots \ldots \ldots \ldots \ldots$ DSTSEADTIPDSTNPSGLPPSVEYEVFL 65 M MSYLRDVATAVALLLDNLCCG ...RPN.... LNNDNEDTIQQTDSTSPVVDPSSSSQSMDSTSVVDAISDSTNPSASFPSVEYDVFL 80

Fig. 9. Signal anchor sequence of L6 is required for full L6 resistance. A, Alignment of the N-terminal sequences preceding the Toll/interleukin-1 receptor (TIR) domain of the flax L6, M, and P2 resistance proteins. Amino acid identities are highlighted in black and similarities in gray. The junction point for domain swaps between the L6 and the M or P2 resistance proteins is arrowed. B, Disease phenotypes of transgenic L6 and P2-L6 flax plants, and the susceptible progenitor, 'Ward', challenged with a strain of flax-rust-expressing AvrL567. The L6 plant shows full resistance characterized by small necrotic flecks, and Ward shows a susceptible phenotype characterized by sporulating pustules releasing orange uredospores. The P2-L6 plants (from two independent transformed lines) show reduced resistance, with some small pustules developing on upper leaves. Upper and lower leaves showing the apical or basal gradient in disease resistance phenotypes are shown below the corresponding whole plants. C, Alignment of the $\mathrm{N}$-terminal sequences preceding the TIR domain of the flax L to L11, M, M1, and M3 resistance proteins. Amino acid identities are highlighted in black and similarities in gray. 
mulated sufficient protein to be detectable by fluorescence microscopy when expressed under the control of the L6 promoter. To test whether the P2-L6 domain swap accumulates less protein than L6 or the M-L6 swap, protein gel blot experiments were conducted on protein extracted from leaves of $N$. benthamiana following agroinfiltration with CIT-tagged L6, M-L6, and P2-L6 constructs expressed under the control of the L6 promoter. The P2-L6 domain swap consistently showed a lower level of protein accumulation compared with M-L6 or L6 alone (Fig. 10D), which could explain the lower level of resistance conferred by P2-L6, although an additional effect on resistance protein function is not excluded by this finding. The protein gel blot also shows that the P2-L6:CIT fusion is intact (Fig. 10D), indicating that the observed cytosolic pattern of fluorescence, although involving a different derivative of GFP, is unlikely to be a consequence of the proteolytic removal of fluorescent protein from the P2-L6 fusion. This is confirmed by the absence of nuclear fluorescence (Fig. 10C), consistent with the inability of YFPv to diffuse freely into the nucleus because the mass of the fusion protein exceeds the nuclear exclusion limit.

\section{DISCUSSION}

Our data (Fig. 1) confirm that the predicted N-terminal membrane attachment domains of the RPP1-WsA, RPP1WsB, RPS5, L6, and M resistance proteins, and the PBS1 protein required for RPS5 resistance, do function in membrane attachment. Our data (Figs. 1, 2, and 8) also suggest that the RPP1-WsB, RPS5, and PBS1 proteins are myristoylated, palmitoylated, and targeted to the PM, consistent with other observations that proteins with the MGCxxS/T myristoylation and palmitoylation motif (or variations thereof) target exclusively to the PM (Batistic et al. 2008). In contrast, the signalanchored RPP1-WsA, L6, and M resistance proteins appear to be targeted to the endomembrane system (Figs. 1 and 3 to 8). Although we did not observe signal anchor localization to the PM in this study, other studies show that signal-anchored plant proteins such as Arabidopsis synaptotagmin 1 and Arabidopsis kinase-associated protein phosphatase can localize to the PM, although it should be noted that synaptotagmin 1 also seems to be located in the ER and exocytic or endocytic vesicles (Schapire et al. 2008) and kinase-associated protein phos- phatase is internalized during endocytosis of client receptorlike protein kinases (Shah et al. 2002).

Our observations (Figs. 1, 2, and 5) support the widely held view that RPS5 and PBS1 are both targeted to the PM and are consistent with the observation that RPS5 and PBS1 form a complex (Ade et al. 2007) that recognizes the PM-targeted AvrPphB effector (Dowen et al. 2009). Similarly, our observation (Figs. 1 and 3) that the RPP1-WsA-30N:GFP fusion protein localizes to both the ER and the GA supports and extends the results of two phase partitioning and sucrose density gradient centrifugation experiments conducted by Weaver and associates (2006), which indicated localization of full-length RPP1-WsA to the ER or the GA. Our data also indicate that, as in L6 and M, the N-terminal signal anchor of RPP1-WsA is sufficient to explain the localization of the full-length protein.

Neither RPM1 nor RPS2 had predicted N-terminal membrane attachment domains despite both being shown previously to be membrane associated. Our data (Fig. 1) indicate that RPM1 does not contain a membrane attachment motif within the N-terminal 100 amino acids of the protein, suggesting that membrane association of RPM1 is mediated by another part of the protein. RPM1 could be indirectly associated with the PM through its interaction with RIN4. This would be consistent with the observation that RPM1 behaves as a peripheral membrane protein (Boyes et al. 1998). In contrast, our data (Fig. 1) show that the N-terminal 30 amino acids of RPS2 do contain targeting information able to direct the RPS2-30N:GFP fusion protein to the PM, albeit incompletely. This region of RPS2 contains cysteine residues at positions 11 and 16 that are potential palmitoylation sites. The N terminus of RPS2 also contains a stretch of hydrophobic amino acids from phenylalanine at position 3 to alanine at position 22 that could potentially function as a signal anchor. Cysteine palmitoylation, signal anchoring, or a combination of both could perhaps enable membrane attachment consistent with the observation that RPS2 behaves as an integral membrane protein (Axtell and Staskawicz 2003).

\section{Mutational analysis of RPS5/PBS1 myristoylation and palmitoylation motifs.}

The $\mathrm{N}$ terminus of RPS5 contains a canonical MGxxxS myristoylation motif, and mutational analysis showed that the conserved glycine and serine residues were both essential for mem-
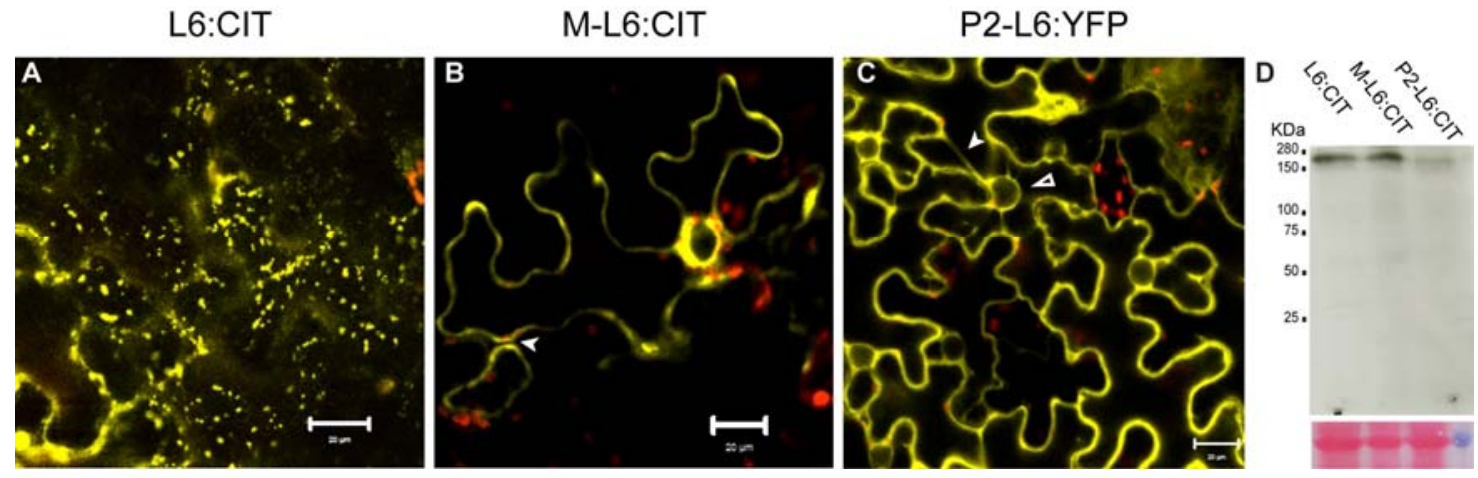

Fig. 10. N-terminal domain swaps between L6, M, and P2 resistance proteins affect both subcellular localization and accumulation of the L6 resistance protein. A, Full-length flax L6 resistance protein fused to the citrine (CIT) variant of green fluorescent protein (GFP) and expressed under the control of the L6 promoter localizes to the Golgi apparatus in epidermal cells of agroinfiltrated Nicotiana benthamiana leaves. Bar = $20 \mu \mathrm{m}$. B, Full-length flax M-L6 domainswap protein fused to the CIT variant of GFP and expressed under the control of the L6 promoter localizes to the tonoplast in epidermal cells of agroinfiltrated $N$. benthamiana leaves. Note the discontinuity in labeling (solid arrowhead) consistent with tonoplast labeling. C, Full-length flax P2-L6 domain-swap protein fused to the Venus yellow fluorescent protein (YFP) variant of GFP and expressed under the control of the Cauliflower mosaic virus 35S promoter localizes to the cytosol in epidermal cells of agroinfiltrated $N$. benthamiana leaves. Cytoslic localization is indicated by the presence of cytoplasmic strands (solid arrowhead) and absence from the nucleus (open arrow head) due to the size (approximately $170 \mathrm{kDa}$ ) of the fusion protein being greater than the nuclear exclusion limit. Bar $=20 \mu \mathrm{m}$. Images in A, B, and C were taken 5 days post agroinfiltration. D, Upper panel shows a gel blot of full-length L6:CIT, M-L6:CIT, and P2-L6:CIT proteins expressed under the control of the L6 promoter in agroinfiltrated N. benthamiana leaves. Protein was extracted 5 days after agroinfiltration and the blot was probed with anti-GFP antibody. Lower panel shows approximately equal protein loading on Ponceau-S-stained membrane. The immunoblot analysis showed similar results in three independent agroinfiltration experiments. 
brane attachment (Fig. 2A). Mutation of the cysteine at position 4 of this motif in RPS5 reduced the efficiency of PM attachment (Fig. 2A), suggesting that palmitoylation of this residue, although not essential, is important to stabilize PM attachment of RPS5. In contrast to RPS5, PBS1 departs from the canonical MGxxxS/T myristoylation motif with a cysteine residue in place of the conserved serine or threonine residue required for myristoylation (i.e., MGxxxC). This variation could lead to an expectation that the subterminal glycine residue of PBS1 is not a good substrate for myristoylation and, in turn, the cysteine residues at positions 3 and 6 are poor substrates for palmitoylation, given that myristoylation is usually a prerequisite for efficient palmitoylation in this context. Nevertheless, localization of the PBS1$100 \mathrm{~N}$ :GFP fusion to the PM is complete (Figs. 1, 2B, 5, and 8). Moreover, mutation of the subterminal glycine to alanine did not eliminate membrane localization of the PBS1-100N:GFP fusion completely (Fig. 2B), suggesting that myristoylation is not absolutely required for PM localization of the PBS1-100N:GFP fusion. The cysteine residues at positions 3 and 6 conform to a $\mathrm{CxxC}$ palmitoylation motif, and mutation of either of these cysteine residues eliminated membrane localization of the PBS1100N:GFP fusion completely (Fig. 2B), suggesting that dual palmitoylation may be required for membrane attachment. The reduced localization of the PBS1-100N:GFP fusion to the PM following mutation of the subterminal glycine to alanine suggests that myristoylation may still be required for efficient palmitoylation. If this is the case, then mutation of the cysteine at position 6 to alanine may, beside a direct effect on palmitoylation, also have had an effect on myristoylation of the subterminal glycine (MGxxxC may not be as good a signal for myristoylation as MGxxxS/T but it may be better than MGxxxA) and, consequently, palmitoylation of the cysteine residue at position 3. Surprisingly, mutation of the phenylalanine residue at position 4 also eliminated membrane localization of the PBS1$100 \mathrm{~N}$ :GFP fusion (Fig. 2B). This is similar to the finding that two phenylalanine residues adjacent to a cluster of three cysteine residues were required for carboxyl-terminal PM attachment of the RIN4 protein (Takemoto and Jones 2005), which is palmitoylated on one or more of the three cysteine residues (Kim et al. 2005). Perhaps the phenylalanine residue in PBS1 facilitates membrane recruitment, thereby compensating for a low myristoylation efficiency.

\section{Mutational analysis of the L6 or M signal anchors.}

Mutational analysis of the 6 amino acid residues immediately adjacent to the $\mathrm{N}$-terminal 15 amino acid residues conserved between L6 and M (Fig. 7) showed that these downstream residues determine the specific membrane to which these signalanchored proteins are targeted. More specifically, mutation of the two cysteine residues (and the adjacent glycine) in this region of $\mathrm{M}$ severely reduced membrane attachment and completely abolished TP targeting of the M-30N:GFP fusion, whereas introduction of the two cysteines (along with the adjacent glycine) into L6 caused almost complete targeting of the L6-30N:GFP fusion to the TP. These two cysteines are potential sites for palmitoylation but treatment with the palmitoylation inhibitor 2-BPA did not affect M-30N:GFP localization, despite being effective for PBS1 (Fig. 8). Thus, both membrane attachment and TP targeting information are most likely determined by the unmodified cysteine residues in the $\mathrm{N}$ terminus of $\mathrm{M}$. Interestingly, the $\mathrm{N}$ terminus of the Arabidopsis calcineurin B-like 6 (CBL6) protein has an amino acid composition very similar to that of $\mathrm{M}$ and has two cysteines in exactly the same position as $\mathbf{M}$ (but no adjacent glycine) and, like $\mathbf{M}$, targets a fluorescent fusion protein to the TP (Batistic et al. 2010). Moreover, the first of these cysteines is conserved in CBL2 and CBL3, which are also localized to the TP (Batistic et al. 2010).

\section{Why are some resistance proteins attached to specific cellular membranes?}

Membrane targeting per se does not seem to be required for RPP1-WsA or L6 function but does seem to contribute to plant disease resistance (Weaver et al. 2006; this work). Our data (Fig. 10D) indicate that membrane localization may stabilize the L6 protein, allowing it to accumulate to higher levels, which may, in turn, contribute to higher levels of defense activation. Localization to the surface of a membrane also imposes a two-dimensional constraint that increases the local concentration of low-abundance proteins relative to the three-dimensional freedom of the cytosol. This could be important if, as evidence suggests (Ade et al. 2007; Bernoux et al. 2011; Gutierrez et al. 2009; Maekawa et al. 2011; Mestre and Baulcombe 2006), dimerization or oligomerization of resistance proteins is an important part of the signaling process. Dimerization, oligomerization, or interaction with other membrane-localized proteins may also be important for protein stability. The reduced resistance provided by the P2-L6 fusion compared with L6 or the M-L6 fusion (Fig. 9) is consistent with these ideas. Moreover, the TIR domain of L6, whose dimerization is critical for autoactive resistance signaling (Bernoux et al. 2011), also requires membrane anchoring for autoactivity (M. Bernoux, unpublished). However, the majority of resistance proteins are not membrane anchored; therefore, this hypothesis fails to explain why L6 and M are membrane anchored when other resistance proteins are not. Nor does it explain why L6 and M are targeted to different membranes. The specificity of membrane targeting may be important if the corresponding effector protein is targeted against a host protein (or other cell component) located on a specific membrane. However, AvrL567 and AvrM accumulate in the host cytosol after transient expression rather than host cell membranes (Rafiqi et al. 2010), and recognition of AvrL567 by L6 and of AvrM by M is direct (Catanzariti et al. 2010; Dodds et al. 2006). Together, these two observations would seem to obviate any need to target L6 and M to the same locations as host proteins targeted by AvrL567 or AvrM.

The tobacco N TIR-NB-LRR resistance protein oligomerizes after recognition of its corresponding ligand (Mestre and Baulcombe 2006) whereas the Arabidopsis RPS5 and tomato Prf CC-NB-LRR proteins seem to occur as oligomers prior to recognition of their corresponding ligands (Ade et al. 2007; Gutierrez et al. 2009). Homodimerization of the L6 TIR domain is required for its resistance signaling function (Bernoux et al, 2011); therefore, like $\mathrm{N}$ in tobacco, the flax L6 and $\mathrm{M}$ proteins may also dimerize after recognition of their corresponding ligands. Because L6 and M are closely related paralogues, it is conceivable that L6 and M could form heterodimers as well as homodimers after interaction with AvrL567 and AvrM. Heterodimerization might reduce the signaling capacity of either or both L6 and M. However, by restricting L6 to one membrane and $M$ to another, the potential for heterodimerization is minimized. Interestingly, all other resistance genes at the multi-allelic L locus (Ellis et al. 1999) and at least two other resistance genes (M1 and M3) at the complex M locus (Lawrence et al. 2010) encode proteins with N-terminal sequences that are identical or nearly identical to that of L6 (Fig. 9C). Therefore, it is highly likely that all the L locus proteins and the M1 and M3 proteins are targeted to the GA and that only the $\mathrm{M}$ protein is targeted to a different location. Thus, avoidance of heterodimerization would be specific to $\mathrm{M}$. Avoidance of heterodimerization might also explain why the Arabidopsis RPP1-WsA TIR-NB-LRR protein is targeted to a different membrane than the closely related RPP1-WsB and RPP1-WsC proteins.

Another possible explanation for membrane localization is suggested by recent observations reported for Arabidopsis 
RPW8.2, a novel signal-anchored resistance protein that confers broad-spectrum resistance to powdery mildew. In uninfected cells, RPW8.2 is targeted to the endomembrane system, possibly to punctate or vesicle-like sites of ER export to the GA (Wang et al. 2007). Remarkably, in infected cells, RPW8.2 is targeted to the extrahaustorial membrane, not only of powdery mildew haustoria but also haustoria of the oomycete pathogen $H$. arabidopsidis (Wang et al. 2009). This suggests that, in the uninfected cell, RPW8.2 is positioned ready for rapid deployment to the extrahaustorial membrane. This raises the intriguing possibility that L6 or M might likewise be poised for mobilization to the extrahaustorial membrane of flax rust haustoria or to endocytic vesicles involved in effector protein delivery and that the GA or TP are merely staging posts. Alternatively, studies using GFP-tagged subcellular organelles have shown accumulation of the GA at the site of haustorium formation (Takemoto et al. 2003), which could have the effect of concentrating L6 at the site of AvrL567 entry.

Thus, there could be at least five strategies underlying membrane attachment of what would otherwise be cytosolic plant disease resistance proteins: i) stabilizing resistance proteins, ii) promoting oligomerization of resistance proteins either before or after effector recognition, iii) facilitating effector recognition by targeting resistance proteins to the same membrane location as a host protein (or other cell component) targeted for attack by the effector, iv) partitioning of resistance proteins to avoid interference between closely related resistance proteins with distinct recognition capabilities, or v) prepositioning for rapid mobilization to the host-pathogen interface.

\section{MATERIALS AND METHODS}

\section{Construction of vectors for plant transformation.}

Fusions of resistance genes RPPI-WsA (Botella et al. 1998), RPPl-WsB (Botella et al. 1998), RPMI (Grant et al. 1995), RPS2 (Bent et al. 1994; Mindrinos et al. 1994), RPP4 (van der Biezen et al. 2002), RPS5 (Warren et al. 1998), RPP8 (McDowell et al. 1998), L6 (Lawrence et al. 1995), $M$ (Anderson et al. 1997), and P2 (Dodds et al. 2001); of PBS1 (Swiderski and Innes 2001) with GFP; and of STtmd with DsRed were generated following polymerase chain reaction (PCR) amplification using mutagenic primers to introduce cloning sites and site-specific mutations as required. Arabidopsis genomic DNA (Col-1 or Ws-0) was used as the template for Arabidopsis resistance genes, and plasmid DNAs carrying flax resistance genes or a derivative of GFP described previously (Benghezal et al. 2000) were used as templates for the flax resistance gene and GFP components, respectively. Vector pDsRed-Express (Clontech, Mountain View, CA, U.S.A.) and STtmd-GFP (Boevink et al. 1998) were used as templates for the DsRed and STtmd components, respectively. All amplified components were cloned and authenticated by sequence analysis. GFP and DsRed fusions were placed under the control of a CaMV 35S promoter and terminator from pCBJ-GFP-hTalin (Takemoto et al. 2003) in binary plant transformation vectors pSLJ7292 (Jones et al. 1992) for generation of transgenic plants or pCBJ352 (Chakrabarti et al. 2009) for agroinfiltration experiments, or in pBluescript $\mathrm{SK}(-)$ (Stratagene, La Jolla, CA, U.S.A.) for bombardment experiments. The citrine fusion to L6 was generated in the pTNL6 $\triangle \mathrm{EB}$ binary vector, which carries a 9-kb flax genomic fragment containing L6 under the control of its own promoter and terminator (Ellis et al. 1999). The $M-L 6$ and P2-L6 chimeric genes were generated by replacing $L 6$ coding sequence in pTNL6 $\triangle \mathrm{EB}$ with $M$ and $P 2$ coding sequences. Details about the construction of these vectors are provided in Supplementary Tables 1 and 2.
Plant growth conditions and plant transformation.

Tobacco (N. tabacum 'Petit Havana') and flax (Linum usitatissimum 'Ward') plants were grown under standard glasshouse conditions at $25^{\circ} \mathrm{C}$ during the day and $18^{\circ} \mathrm{C}$ at night, and leaves were harvested from 4- to 8-week-old plants for particle bombardment experiments. Arabidopsis plants (Arabidopsis thaliana ecotype Columbia) were grown at $21^{\circ} \mathrm{C}$ with $16 \mathrm{~h}$ of light $\left(50 \mu \mathrm{mol}\right.$ of photons $\left.\mathrm{m}^{-2} \mathrm{~s}^{-1}\right)$ per day before transformation or particle bombardment. Transformation of Arabidopsis was performed by the floral dip method (Clough and Bent 1998) using Agrobacterium tumefaciens AGL-1 (Lazo et al. 1991). Screening of Arabidopsis transformants was performed as described previously (Takemoto et al. 2003).

A. tumefaciens GV3101(pMP90) was used to transform the flax line Ward as described by Anderson and associates (1997), except that the selective agent spectinomycin was used at 50 $\mu \mathrm{g} / \mathrm{ml}$. Single-copy transformants were selected by DNA gel blot hybridization. Inoculations with rust strain $\mathrm{CH} 5(84)$ were performed as described by Lawrence and associates (1981) and agroinfiltrations with AvrL567 were carried as described by Dodds and associates (2004).

Bombardments were carried out using a Bio-Rad PDS1000/He Biolistic Particle Delivery System (Bio-Rad, Hercules, CA, U.S.A.) according to the manufacturer's instructions, and no fewer than 20 transformed epidermal cells were monitored for each fusion construct by confocal laser-scanning microscopy $6 \mathrm{~h}$ after bombardments. For co-bombardment experiments, $400 \mathrm{ng}$ of each vector containing GFP fusions, DsRed fusion, or the AvrRpt2 gene were used to coat $1.25 \mathrm{mg}$ of 1$\mu \mathrm{m}$-diameter gold particles in a $25-\mu \mathrm{l}$ suspension.

For agroinfiltrations, cultures of A. tumefaciens GV3101 (pMP90) carrying binary vectors were grown overnight at $28^{\circ} \mathrm{C}$ in liquid YEP medium with appropriate antibiotics and $20 \mu \mathrm{M}$ acetosyringone. The overnight cultures were centrifuged at $5,000 \times g$ for $10 \mathrm{~min}$ at room temperature and resuspended to an optical density at $600 \mathrm{~nm}$ of 1 in $10 \mathrm{mM}$ MES (pH 5.6), 2\% (wt/vol) sucrose, $1 \%$ (wt/vol) glucose, and $200 \mu \mathrm{M}$ acetosyringone. Cultures were incubated for at least $2 \mathrm{~h}$ at $18^{\circ} \mathrm{C}$ with gentle shaking before being used for infiltration. For N. benthamiana infiltrations, cultures carrying resistance protein constructs were mixed 1:1 with a culture carrying a Tomato bushy stunt virus p19 suppressor of silencing construct able to suppress transgene silencing (Voinnet et al. 2003).

\section{Confocal laser-scanning microscopy.}

Confocal fluorescence images were recorded on a Leica microscope with a Leica SP2 confocal system (Leica, Sydney, Australia), using $\times 40$ and $\times 63 \mathrm{C}$-Apochromat water-immersion objectives. The 488-nm laser line with appropriate emission filters was used to image GFP and DsRed and the 514-nm laser line was used to image citrine. Images of GFP and DsRed fluorescence shown in Figures 1 to 4, and 7 are projections of optical sections taken at $1-\mu \mathrm{m}$ intervals from the outer epidermal wall through to a position just above the cortical cytoplasm adjacent to the inner periclinal wall of the epidermal cell. The images were stored as TIF files and processed with Canvas 8 software (Deneba Systems Inc., Miami). Images of GFP citrine and YFPv fluorescence shown in Figures 5, 6, 8, and 10 are 1$\mu \mathrm{m}$ sections taken at various positions within the cell. The images were stored as TIF files and processed using Photoshop 7 software (Adobe Systems Inc., San Jose, CA, U.S.A.).

\section{Immunoblot analysis.}

Tissue from agroinfiltrated $N$. benthamiana leaves was frozen and ground in liquid nitrogen and resuspended in either 50 $\mathrm{mM}$ Tris- $\mathrm{Cl}$ (pH 7.5), 5 mM EDTA (pH 8.0), $10 \%$ glycerol, or $150 \mathrm{mM} \mathrm{NaCl}, 20 \mathrm{mM}$ Tris-Cl (pH 6.5), 1 mM EDTA, 1\% Tri- 
ton $\mathrm{X}-100,0.1 \% \beta$-mercaptoethanol, and $1 \mathrm{mM}$ PMSF (used for the blot shown in Figure 10D). Extraction buffer was supplemented with Roche Complete Protease Inhibitor Cocktail (Roche Diagnostics Ltd., Mannheim, Germany). The samples were then centrifuged to remove cellular debris before incubation for $10 \mathrm{~min}$ at $70^{\circ} \mathrm{C}$ in the presence of Laemmli buffer with $\beta$-mercaptoethanol (Laemmli 1970). Samples were separated by gradient $(4-12 \%)$ sodium dodecyl sulfate polyacrylamide gel electrophoresis and electroblotted to Hybond C-extra nitrocellulose membranes (Amersham Biosciences, Piscataway, NJ, U.S.A.). Citrine fusion proteins were detected using a SuperSignal West Pico chemiluminescence kit (Pierce, Rockford, IL, U.S.A.), an anti-GFP primary antibody (Roche Diagnostics Ltd.), and an anti-mouse secondary antibody conjugated to horseradish peroxidase (Bio-Rad).

\section{ACKNOWLEDGMENTS}

Funding for part of this work was provided by the Australian Research Council (Discovery Project grants DP0771374 and DP1093850). We thank S. Dinesh-Kumar of Yale University for provision of the citrine variant of GFP and L. Deslandes for provision of the 35S YFPv Gateway binary vector.

\section{LITERATURE CITED}

Ade, J., DeYoung, B. J., Golstein, C., and Innes, R. W. 2007. Indirect activation of a plant nucleotide binding site-leucine-rich repeat protein by a bacterial protease. Proc. Natl. Acad. Sci. U.S.A. 104:2531-2536.

Anderson, P. A., Lawrence, G. J., Morrish, B. C., Ayliffe, M. A., Finnegan, E. J., and Ellis, J. G. 1997. Inactivation of the flax rust resistance gene $M$ associated with loss of a repeated unit within the leucine-rich repeat coding region. Plant Cell 9:641-651.

Andriotis, V. M. E., and Rathjen, J. P. 2006. The Pto kinase of tomato, which regulates plant immunity, is repressed by its myristoylated $\mathrm{N}$ terminus. J. Biol. Chem. 281:26578-26586.

Axtell, M. J., and Staskawicz, B. J. 2003. Initiation of RPS2-specified disease resistance in Arabidopsis is coupled to the AvrRpt2-directed elimination of RIN4. Cell 112:369-377.

Batistic, O., Sorek, N., Schültke, S., Yalovsky, S., and Kudla, J. 2008. Dual fatty acyl modification determines the localization and plasma membrane targeting of CBL/CIPK Ca ${ }^{2+}$ signaling complexes in Arabidopsis. Plant Cell 20:1346-1362.

Batistic, O., Waadt, R., Steinhorst, L., Held, K., and Kudla, J. 2010. CBLmediated targeting of CIPKs facilitates the decoding of calcium signals emanating from distinct cellular stores. Plant J. 61:211-222.

Benghezal, M., Wasteneys, G. O., and Jones, D. A. 2000. The C-terminal dilysine motif confers ER localization to type I membrane proteins in plants. Plant Cell 12:1179-1201.

Bent, A. F., Kunkel, B. N., Dahlbeck, D., Brown, K. L., Schmidt, R., Giraudat, J., Leung, J., and Staskawicz, B. J. 1994. RPS2 of Arabidopsis thaliana: a leucine-rich repeat class of plant disease resistance genes. Science 265:1856-1860.

Bernoux, M., Ve, T., Williams, S., Warren, C., Hatters, D., Valkov, E., Zhang, X., Ellis, J. G., Kobe, B., and Dodds, P. N. 2011. Structural and functional analysis of a plant resistance protein TIR domain reveals interfaces for self-association, signaling and autoregulation. Cell Host Microbe 9:200-211.

Boevink, P., Oparka, K., Santa Cruz, S., Martin, B., Betteridge, A., and Hawes, C. 1998. Stacks on tracks: the plant Golgi apparatus traffics on an actin/ER network. Plant J. 15:441-447.

Botella, M. A., Parker, J. E., Frost, L. N., Bittner-Eddy, P. D., Beynon, J. L., Daniels, M. J., Holub, E. B., and Jones, J. D. G. 1998. Three genes of the Arabidopsis RPP1 complex resistance locus recognize distinct Peronospora parasitica avirulence determinants. Plant Cell 10:18471860.

Boyes, D. C., Nam, J., and Dangl, J. L. 1998. The Arabidopsis thaliana RPM1 disease resistance gene product is a peripheral plasma membrane protein that is degraded coincident with the hypersensitive response. Proc. Natl. Acad. Sci. U.S.A. 95:15849-15854.

Catanzariti, A. M., Dodds, P. N., Ve, T., Kobe, B., Ellis, J. G., and Staskawicz, B. J. 2010. The AvrM effector from flax rust has a structured C-terminal domain and interacts directly with the $\mathrm{M}$ resistance protein. Mol. Plant-Microbe Interact. 23:49-57.

Chakrabarti, A., Panter, S. N., Harrison, K., Jones, J. D. G., and Jones, D.
A. 2009. Regions of the Cf-9B disease resistance protein able to cause spontaneous necrosis in Nicotiana benthamiana lie within the region controlling pathogen recognition in tomato. Mol. Plant-Microbe Interact. $22: 1214-1226$

Clough, S. J., and Bent, A. F. 1998. Floral dip: a simplified method for Agrobacterium-mediated transformation of Arabidopsis thaliana. Plant J. 16:735-743.

Day, B., Dahlbeck, D., Huang, J., Chisholm, S. T., Li, D., and Staskawicz, B. J. 2005. Molecular basis for the RIN4 negative regulation of RPS2 disease resistance. Plant Cell 17:1292-1305.

de Vries, J. S., Andriotis, V. M. E., Wu, A.-J., and Rathjen, J. P. 2006. Tomato Pto encodes a functional $N$-myristoylation motif that is required for signal transduction in Nicotiana benthamiana. Plant J. 45:31-45.

Dodds, P. N., and Rathjen, J. P. 2010. Pathogen perception and responses in plant immunity. Nat. Rev. Genet. 11:539-548.

Dodds, P. N., Lawrence, G. J., and Ellis, J. G. 2001. Six amino acid changes confined to the leucine-rich repeat beta-strand/beta-turn motif determine the difference between the $P$ and $P 2$ rust resistance specificities in flax. Plant Cell 13:163-178.

Dodds, P. N., Lawrence, G. J. , Catanzariti, A.-M., Ayliffe, M., and Ellis J. G. 2004. The Melampsora lini AvrL567 avirulence genes are expressed in haustoria and their products are recognised inside plant cells. Plant Cell 16:755-768.

Dodds, P. N., Lawrence, G. J., Catanzariti, A.-M., Teh, T., Wang, C. I., Ayliffe, M. A., Kobe, B., and Ellis, J. G. 2006. Direct protein interaction underlies gene-for-gene specificity and coevolution of the flax resistance genes and flax rust avirulence genes. Proc. Natl. Acad. Sci. U.S.A. 103:8888-8893.

Dowen, R. H., Engel, J. L., Shao, F., Ecker, J. R., and Dixon, J. E. 2009. A family of bacterial cysteine protease type III effectors utilizes acylationdependent and -independent strategies to localize to plasma membranes. J. Biol. Chem. 284:15867-15879.

Ellis, J. G., Lawrence, G. J., Luck, J. E., and Dodds, P. N. 1999. Identification of regions in alleles of the flax rust resistance gene $L$ that determine differences in gene-for-gene specificity. Plant Cell 11:495-506.

Grant, M. R., Godiard, L., Straube, E., Ashfield, T., Lewald, J., Sattler, A, Innes, R. W., and Dangl, J. L. 1995. Structure of the Arabidopsis RPMI gene enabling dual specificity disease resistance. Science 269:843-846.

Griesbeck, O., Baird, G. S., Campbell, R. E., Zacharias, D. A., and Tsien, R. Y. 2001. Reducing the environmental sensitivity of yellow fluorescent protein. Mechanism and applications. J. Biol. Chem. 276:2918829194.

Gunning, B. E. S. 1998. The identity of mystery organelles in Arabidopsis plants expressing GFP. Trends Plant Sci. 3:417.

Gutierrez, J. R., Balmuth, A. L., Ntoukakis, V., Mucyn, T. S., GimenezIbanez, S., Jones, A. M. E., and Rathjen, J. P. 2009. Prf immune complexes of tomato are oligomeric and contain multiple Pto-like kinases that diversify effector recognition. Plant J. 61:507-518.

Holt, B. F., III, Belkhadir, Y., and Dangl, J. L. 2005. Antagonistic control of disease resistance protein stability in the plant immune system. Science 309:929-932.

Johnson, D. R., Bhatnagar, R. S., Knoll, L. J., and Gordon, J. I. 1994. Genetic and biochemical studies of protein N-myristoylation. Annu. Rev. Biochem. 63:869-914.

Jones, J. D. G., and Dangl, J. L. 2006. The plant immune system. Nature 16:323-329.

Jones, J. D. G., Shlumukov, L., Carland, F., English, J., Scofield, S. R., Bishop, G. J., and Harrison, K. 1992. Effective vectors for transformation, expression of heterologous genes, and assaying transposon excision in transgenic plants. Transgenic Res. 1:285-297.

Kim, H. S., Desveaux, D., Singer, A. U., Patel, P., Sondek, J., and Dangl, J. L. 2005. The Pseudomonas syringae effector AvrRpt2 cleaves its Cterminally acylated target, RIN4, from Arabidopsis membranes to block RPM1 activation. Proc. Natl. Acad. Sci. U.S.A. 102:6496-64501.

Laemmli, U. K. 1970. Cleavage of structural proteins during the assembly of the head of bacteriophage T4. Nature 227:680-685.

Lawrence, G. J., Mayo, G. M. E., and Shepherd, K. W. 1981. Interactions between genes controlling pathogenicity in the flax rust fungus. Phytopathology 71:12-19.

Lawrence, G. J., Finnegan, E. J., Ayliffe, M. A., and Ellis, J. G. 1995. The L6 gene for flax rust resistance is related to the Arabidopsis bacterial resistance gene RPS2 and the tobacco viral resistance gene $N$. Plant Cell 7:1195-1206.

Lawrence, G. J., Anderson, P. A., Dodds, P. N., and Ellis J. G. 2010. Relationships between rust resistance genes at the $M$ locus in flax. Mol. Plant Pathol. 11:19-32.

Lazo, G. R., Stein, P. A,.., and Ludwig, R. A. 1991. A DNA transformationcompetent Arabidopsis genomic library in Agrobacterium. Bio/Technology 9:963-967.

Maekawa, T, Cheng, W., Spiridon, L. N., Töller, A., Lukasik, E., Saijo, Y., 
Liu, P., Shen, Q.-H., Micluta, M. A., Somssich, I. E., Takken, F. L. W., Petrescu, A.-J., Chai, J., and Schulze-Lefert, P. 2011. Coiled-coil domain-dependent homodimerization of intracellular barley immune receptors defines a minimal functional module for triggering cell death. Cell Host Microbe 9:187-199.

McDowell, J. M., Dhandaydham, M., Long, T. A., Aarts, M. G. M., Goff, S., Holub, E. B., and Dangl, J. L. 1998. Intragenic recombination and diversifying selection contribute to the evolution of downy mildew resistance at the RPP8 locus of Arabidopsis. Plant Cell 10:1861-1874.

Mestre, P., and Baulcombe, D. C. 2006. Elicitor-mediated oligomerization of the tobacco $\mathrm{N}$ disease resistance protein. Plant Cell 18:491501

Mindrinos, M., Katagiri, F., Yu, G. L., and Ausubel, F. M. 1994. The A. thaliana disease resistance gene RPS2 encodes a protein containing a nucleotide-binding site and leucine-rich repeats. Cell 78:1089-1099.

Nagai, T., Ibata, K., Park, E. S., Kubota, M., Mikoshiba, K., and Miyawaki, A. 2002. A variant of yellow fluorescent protein with fast and efficient maturation for cell-biological applications. Nat. Biotechnol. 20:87-90.

Nimchuk, Z., Marois, E., Susanne Kjemtrup, S., Leister, R. T., Katagiri, F., and Dangl, J. L. 2000. Eukaryotic fatty acylation drives plasma membrane targeting and enhances function of several type III effector proteins from Pseudomonas syringae. Cell 101:353-363.

Rafiqi, M., Bernoux, M., Ellis, J. G., and Dodds, P. N. 2009. In the trenches of plant pathogen recognition: role of NB-LRR proteins. Semin. Cell Dev. Biol. 20:1017-1024.

Rafiqi, M., Gan, P. H. P., Ravensdale, M., Lawrence, G. J., Ellis, J. G., Jones, D. A., Hardham, A. R., and Dodds, P. N. 2010. Internalization of flax rust avirulence proteins into plant cells can occur in the absence of the pathogen. Plant Cell 22:2017-2032.

Reisen, D., Marty, F., and Leborgne-Castel, N. 2005. New insights into the tonoplast architecture of plant vacuoles and vacuolar dynamics during osmotic stress. BMC Plant Biol. 5:13.

Ritzenthaler, C., Nebenführ, A., Movafeghi, A., Stussi-Garaud, C., Behnia, L., Pimpl, P., Staehelin, L. A., and Robinson, D. G. 2002. Reevaluation of the effects of brefeldin A on plant cells using tobacco Bright Yellow 2 cells expressing Golgi-targeted green fluorescent protein and COPI antisera. Plant Cell 14:237-261

Saito, C., Ueda, T., Abe, H., Wada, Y., Kuroiwa, T., Hisada, A., Furuya, M., and Nakan, A. 2002. A complex and mobile structure forms a distinct subregion within the continuous vacuolar membrane in young cotyledons of Arabidopsis. Plant J. 29:245-255.

Schapire, A. L., Voigt, B., Jasik, J., Rosado, A., Lopez-Cobollo, R., Menzel, D., Salinas, J., Mancuso, S., Valpuesta, V., Balusk, F., and Botella, M. A. 2008. Arabidopsis synaptotagmin 1 is required for the maintenance of plasma membrane integrity and cell viability. Plant Cell 20:3374-3388.

Shah, K., Russinova, E., Gadella, T. W. J., Jr., Willemse, J., and De Vries, S. C. 2002. The Arabidopsis kinase-associated protein phosphatase controls internalization of the somatic embryogenesis receptor kinase 1 . Genes Dev. 16:1707-1720.

Shahinian, S., and Silvius, J. R. 1995. Doubly-lipid-modified protein sequence motifs exhibit long-lived anchorage to lipid bilayer membranes. Biochemistry 34:3813-3822.

Shan, L., Thara, V. K., Martin, G. B., Zhou, J.-M., and Tang, X. 2000. The Pseudomonas AvrPto protein is differentially recognized by tomato and tobacco and is localized to the plant plasma membrane. Plant Cell 12:2323-2337.

Swiderski, M. R., and Innes, R. W. 2001. The Arabidopsis PBS1 resistance gene encodes a member of a novel protein kinase subfamily. Plant $\mathbf{J}$ 26:101-112.

Takemoto, D., and Jones, D. A. 2005. Membrane release and destabilization of Arabidopsis RIN4 following cleavage by Pseudomonas syringae AvrRpt2. Mol. Plant-Microbe Interact. 18:1258-1268.

Takemoto, D., Jones, D. A., and Hardham, A. R. 2003. GFP-tagging of cell components reveals the dynamics of subcellular re-organization in response to infection of Arabidopsis by oomycete pathogens. Plant J. 33:775-792.

van der Biezen, E. A., Freddie, C. T., Kahn, K., Parker, J. E., and Jones, J. D. G. 2002. Arabidopsis RPP4 is a member of the RPP5 multigene family of TIR-NB-LRR genes and confers downy mildew resistance through multiple signaling components. Plant J. 29:439-451.

Voinnet, O., Rivas, S., Mestre, P., and Baulcombe, D. 2003. An enhanced transient expression system in plants based on suppression of gene silencing by the p19 protein of tomato bushy stunt virus. Plant J. 33:949-956.

Wang, W., Devoto, A., Turner, J. G., and Xiao, S. 2007. Expression of the membrane-associated resistance protein RPW8 enhances basal defense against biotrophic pathogens. Mol. Plant-Microbe Interact. 20:966-976.

Wang, W., Wen, Y., Berkey, R., and Xiao, S. 2009. Specific targeting of the Arabidopsis resistance protein RPW8.2 to the interfacial membrane encasing the fungal haustorium renders broad-spectrum resistance to powdery mildew. Plant Cell 21:2898-2913.

Warren, R. F., Henk, A., Mowery, P., Holub, E., and Innes, R. W. 1998. A mutation within the leucine-rich repeat domain of the Arabidopsis disease resistance gene RPS5 partially suppresses multiple bacterial and downy mildew resistance genes. Plant Cell 10:1439-1452.

Weaver, L. M., Swiderski, M. R., Li, Y., and Jones, J. D. G. 2006. The Arabidopsis thaliana TIR-NB-LRR R-protein, RPP1A; protein localization and constitutive activation of defense by truncated alleles in tobacco and Arabidopsis. Plant J. 47:829-840. 